\title{
Particle dynamics in 3-D reconnecting current sheets in the solar atmosphere
}

\author{
C. Efthymiopoulos ${ }^{1}$, C. Gontikakis ${ }^{1}$, and A. Anastasiadis ${ }^{2}$ \\ 1 Research Center for Astronomy and Applied Mathematics, Academy of Athens, Soranou Efessiou 4, 11527 Athens, Greece \\ e-mail: cefthim@cc.uoa.gr; cgontik@cc.uoa.gr \\ 2 National Observatory of Athens, Institute for Space Applications \& Remote Sensing, 15236 Palaia Penteli, Greece \\ e-mail: anastasi@space.noa.gr
}

Received 1 November 2004 / Accepted 15 July 2005

\section{ABSTRACT}

The orbits of charged particles (electrons and protons), in a Harris-type 3D field topology of a reconnecting current sheet (RCS), are analyzed by dynamical systems methods. The focus is on values of the magnetic and electric fields relevant to RCSs in the solar atmosphere. First, a perturbative form of the equations of motion is used to determine the stability perpendicularly to the plane of reconnection, which is crucial in the efficiency of the RCS as an accelerator. The problem is shown to correspond to a case of "parametric resonance". The orbits are then studied with the complete form of the equations of motion. These can be reduced to a two degrees of freedom Hamiltonian nonlinear system by exploiting the existence of an additional integral of motion besides the energy. The orbits are studied analytically by normal form theory. Regular and chaotic orbits are identified by the use of appropriate Poincaré surfaces of section. The kinetic energy gain for escaping particles is calculated as a function of the initial conditions of injection of an orbit in the sheet. Formulae relating the kinetic energy gain to the physical parameters of the sheet and the initial conditions of the orbits are given both for electrons and protons.

Key words. acceleration of particles - Sun: magnetic fields - chaos

\section{Introduction}

One of the most puzzling problems in Astrophysics, for the past several decades, has been to understand how the magnetic field in the solar and stellar atmospheres is able to accelerate particles to relativistic velocities and, also, to heat the plasma to temperatures that can reach tens of million degrees to form what we know as coronas. Scientists expect the answer to come from the solar corona which, being closest to us, is observed in most detail.

The mechanism which provides the magnetic energy is magnetic reconnection and it is based on topologies involving magnetic fields with opposite orientation, which come close together within very small distances (e.g. Priest \& Forbes 2000). Theoretical models have been developed to study steady reconnecting topologies, called 'reconnecting current sheets'. Such topologies are typically characterized by the presence of an electric field, normal to the reconnecting magnetic field component. The electric field is equal to $-\boldsymbol{v} \times \boldsymbol{B}$, where $\boldsymbol{v}$ is the velocity of the plasma bulk flow across the sheet and $B$ is the value of the reconnecting magnetic field. As the plasma is flowing into the sheet, the electric field accelerates the charged particles. For super-Dreicer electric fields, the orbits of such particles can be considered as effectively collisionless, thus they can be studied by orbital theory.

There is a variety of scaling formulae in the literature relating the kinetic energy gain of accelerated particles (electrons or ions) as well as the form of the final kinetic distributions to the physical parameters of the reconnecting region (e.g. Speiser 1965; Bulanov 1980; Martens 1988; Martens \& Young 1990; Burkhart et al. 1990; Deeg et al. 1991; Bruhwiler \& Zweibel 1992; Moses et al. 1993; Litvinenko 1996; Vekstein \& Browning 1997; Browning \& Vekstein 2001; Craig \& Litvinenko 2002; Dalla \& Browning 2005). The result seems to depend on the particular topology of the reconnection site (e.g. simple current sheet, X-type, spine), the position of the particles' injection and the relative strengths of the electric and magnetic fields within it. A common question of many studies concerns what should be the values of the different magnetic field components (reconnecting, transverse, longitudinal) in various topologies, so that electrons and protons with initial velocities close to thermal can be accelerated to kinetic energies of tens or hundreds of $\mathrm{keV}$, for electrons, and tens of $\mathrm{MeV}$ for protons. Many authors have also presented simulations with particles for various choices of the RCS model and initial kinetic distribution (e.g. Kliem 1994; Fletcher \& Petkaki 1997; Petkaki \& MacKinnon 1997; Mori et al. 1998; Heerikhuisen et al. 2002; Nodes et al. 2003; Hamilton et al. 2003, 2005; Zharkova \& Gordovskyy 2004, 2005).

A suitable way to distinguish which phenomena are generic and which are model-specific is by a precise theoretical study of the orbits in various reconnecting topologies. Such is the purpose of the present paper. We choose the simplest possible 
reconnecting topology, a Harris-type configuration, and study the orbits in it by methods obtained from the theory of dynamical systems.

Starting with Speiser (1965), the theoretical study of orbits within Harris- type RCSs has shown that, when there is no magnetic field component normal to the reconnection plane, the particles are trapped in the current sheet forever, being accelarated all along its length. In the case, however, of a non-zero normal component of the magnetic field, the resulting Lorentz force carries the particles out of the accelarator quickly and the gain of kinetic energy is limited. The so obtained kinetic energy is insufficient to explain observed kinetic energies of the particles in solar flares (Speiser \& Lyons 1984). Speiser reached these conclusions by an analysis based on perturbation theory. Later on, Cowley (1978) gave rigorous results based on the existence of first integrals of the equations of motion (adiabatic invariants). Chen \& Palmadesso (1986) showed that for a normal component large enough, there are both mirrortype (trapped) and chaotically scattered (untrapped) orbits. However, in the case of solar RCSs, the escaping particles leave the sheet before being efficiently accelerated by the electric field (Litvinenko 1996). Büchner \& Zelenyi (1989) explicitly calculated the adiabatic invariants, and showed that the scattering of particles in chaotic orbits can be described by a nonlinear symplectic mapping similar to the Chririkov standard-map model (Chirikov 1979).

Following the above studies, the orbits of particles in various 2-D field topologies were studied by means of dynamical systems' methods by a number of researchers mainly in the field of magnetospheric physics (see Chen 1992, and references therein for a comprehensive review, and Kliem 1994). On the other hand, the orbits of particles in 3-D topologies involving a component of the magnetic field parallel to the electric field are less well studied. This is called a "longitudinal component". Büchner \& Zelenyi (1991) provided a heuristic criterion for the onset of chaoticity, corresponding essentially to the curvature of the magnetic field lines. Zhu \& Parks (1993) gave analytical expressions for the orbits when the longitudinal component is non-zero while the normal component is equal to zero. The most general (and realistic) case, where all three components of the magnetic field are present, was studied in a series of papers by Litvinenko \& Somov (1993), and Litvinenko (1993, 1996). The main conclusion of these studies is that a large value of the longitudinal component forces the untrapped particles to remain within the sheet for a transient period of time which is long enough so as to allow for the electric field to accelerate the particles to observed values of their kinetic energies. For a main reconnecting field of order $\sim 100 \mathrm{G}$, a normal component $\sim 1 \mathrm{G}$, and an electric field $\sim 10 \mathrm{~V} / \mathrm{cm}$, the longitudinal field should be $10 \sim 100 \mathrm{G}$ to explain final kinetic energies of $10 \sim 100 \mathrm{keV}$ for electrons.

Recent theoretical models (Anastasiadis et al. 1997, 2004; Vlahos et al. 2004) consider the presence of multiple current sheets in complex solar active regions. These models use as an input the kinetic energy gain of particles per single particle RCS interaction. The kinetic energy gain, as a function of the physical parameters of the RCS, is an important and crucial point in the development of such models.
The present paper reports the results from a detailed exploration of the orbital dynamics in a simple 3D model RCS. After describing the model in Sect. 2, Sect. 3 makes a study of the stability problem in a perturbed form of the equations of motion in view of the theory of parametric resonance (e.g. Arnold 1978). This allows us to find an improvement of the criterion of stability for electron orbits given in Litvinenko and Somov (1993). Section 4 gives a theoretical analysis of the orbits by reducing the problem to a a two degrees of freedom nonlinear Hamiltonian model. The main tools used in the theoretical analysis are the normal form method and the method of a Poincaré surface of section. Section 5 presents the main numerical simulations as well as the determination of the kinetic energy gain for escaping particles, as a function of the position of injection and initial kinetic energy. These quantities are calculated numerically for protons and electrons and they are compared with analytical formulae derived from the preceding theoretical analysis. Section 6 summarizes our main conclusions.

\section{The model}

The same simple RCS topology is considered as in Litvinenko \& Somov (1993) and Litvinenko (1996). The current sheet configuration is shown in Fig. 1. The magnetic and electric fields are:

$$
\begin{aligned}
& \boldsymbol{E}=(0,0, E) \\
& \boldsymbol{B}=\left(-y / a, \xi_{\perp}, \xi_{\|}\right) B_{0} \quad \text { for }|y| \leq a \\
& \boldsymbol{B}=\left(-\operatorname{sgn}(y), \xi_{\perp}, \xi_{\|}\right) B_{0} \quad \text { for }|y|>a
\end{aligned}
$$

where $B_{0}$ is a typical measure of the magnetic field in a reconnecting region, $\xi_{\perp}, \xi_{\|}, E$ are constants, and $\operatorname{sgn}(y)=y /|y|$. Thus, the magnetic field has constant measure outside the sheet. This form of the magnetic field in the $x$-axis is a simplification of the Harris configuration $B_{0} \tanh (y / a)$. In fact, all the orbits in the present paper are integrated up to $|y|=a$, while, when an orbit crosses the value $|y|=a$, it is considered as escaping the sheet. Thus, the details of the magnetic field outside the sheet are not important in the analysis below.

A charged particle with mass $m$ and charge $q$ will be under the effect of a Lorentz force, and the non-relativistic equation of motion is

$m \frac{\mathrm{d} \boldsymbol{v}}{\mathrm{d} t}=q(\boldsymbol{E}+\boldsymbol{v} \times \boldsymbol{B})$.

Equation (2), can be analysed in three scalar differential equations in dimensionless form, if the half-thickness $a$ is the unit of length and the inverse gyrofrequency $\omega_{B}^{-1}=m / q B_{0}$ is the unit of time.

$$
\begin{aligned}
& \frac{\mathrm{d}^{2} x}{\mathrm{~d} t^{2}}=\xi_{\|} \frac{\mathrm{d} y}{\mathrm{~d} t}-\xi_{\perp} \frac{\mathrm{d} z}{\mathrm{~d} t} \\
& \frac{\mathrm{d}^{2} y}{\mathrm{~d} t^{2}}=-\xi_{\|} \frac{\mathrm{d} x}{\mathrm{~d} t}-y \frac{\mathrm{d} z}{\mathrm{~d} t} \\
& \frac{\mathrm{d}^{2} z}{\mathrm{~d} t^{2}}=\epsilon+\xi_{\perp} \frac{\mathrm{d} x}{\mathrm{~d} t}+y \frac{\mathrm{d} y}{\mathrm{~d} t}
\end{aligned}
$$




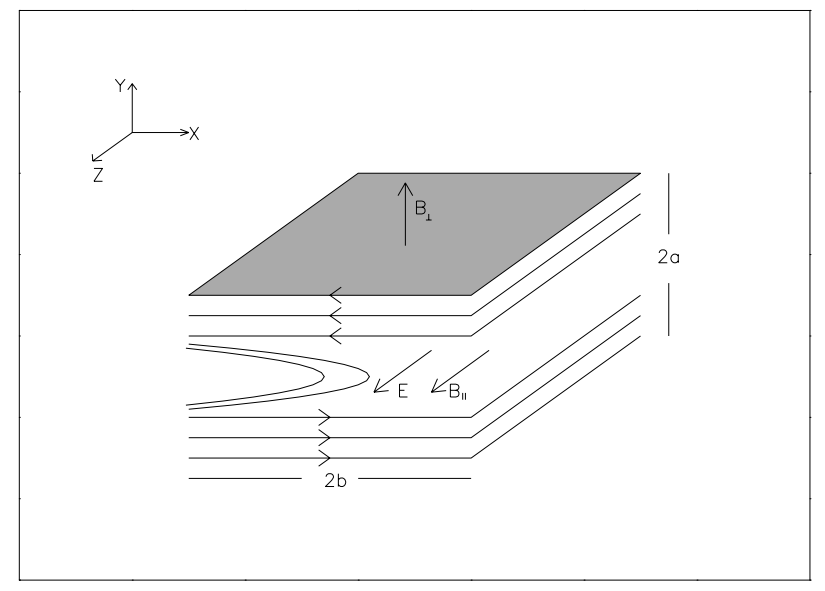

Fig. 1. The topology of the magnetic and electric fields in the model current sheet.

In these equations the dimensionless electric field is given as $\epsilon=m E /\left(a q B_{0}^{2}\right)$, and the quantities $\xi_{\perp}$ and $\xi_{\|}$are defined in Eq. (1). The above equations hold for a positive charge with the orientation of the axes and fields as shown in Fig. 1, or for a negative charge with either the orientation of the $y$ and $z$ axes reversed, or the orientation of the magnetic and electric fields reversed.

The units of length and time in Eqs. (3)-(5), as well as the values of the dimensionless parameters $\xi_{\|}, \xi_{\perp}$ and $\epsilon$ are determined by the values of the main component of the magnetic field $B_{0}$, the electric field $E$, the half-thickness of the sheet $a$, and the nature of the charged particle (electron or proton). Typical values of $a, B_{0}$, and $E$ for solar flares are induced by observations, and/or analysis of the consistency of the plasma parameters with the induced fields (e.g. Martens 1988; Martens \& Young 1990; Somov 1992; Litvinenko 1996, and references therein). These values are $\sim 100 \mathrm{~cm}$ for $a, 100 \sim 200 \mathrm{G}$ for $B_{0}$, $1 \sim 10 \mathrm{~V} / \mathrm{cm}$ for $E$, and $0.1 \sim 1 \mathrm{G}$ for $B_{\perp}$. The electric field is super-Dreicer by about five orders of magnitude, thus the study of individual orbits is justified. On the other hand, the longitudinal component of the magnetic field $B_{\|}$can vary from zero to a value as large as that of the main component $B_{0}$ (the ratio $B_{\|} / B_{0}$ determines the angle of contact of the reconnecting field lines in two-loop models). This parameter is crucial in the problem of particle acceleration because a value $B_{\|} \simeq B_{0}$ is required to explain the observed kinetic energies, at least of electrons, in solar flares (Litvinenko 1996). A non-zero value of $B_{\|}$is also supported by vector magnetic field measurements (e.g. Hagyard 1990) and by observations of the geometry of flare ribbons through their footpoints' motions (Fletcher et al. 2004). In the present paper we examine the cases of a moderate value $\left(=0.1 B_{0}\right)$ and of a large value $\left(=B_{0}\right)$ for $B_{\|}$.

If the unit of the magnetic field is taken equal to $\langle B\rangle=$ $100 \mathrm{G}$, the scaled equations $(4,5,6)$ correspond to electron motion for a choice of time unit $\langle t\rangle=\omega_{B}^{-1}=5.8 \times 10^{-10} \mathrm{~s}$. The length unit is set equal to $\langle L\rangle=a=0.58 \mathrm{~m}$, yielding the velocity unit $\langle v\rangle=10^{9} \mathrm{~m} / \mathrm{s}$ and the energy unit $\langle E\rangle=m_{\mathrm{e}}\langle v\rangle^{2}=$ $5.7 \times 10^{3} \mathrm{keV}$ (the velocities considered in the present paper are at most $v=0.1$, with typical values one order of magnitude smaller, i.e., they can be treated by non-relativistic equations of motion). With the above choice, the electric field unit is $\langle\mathcal{E}\rangle=$ $a B_{0}^{2} q_{\mathrm{e}} / m_{\mathrm{e}}=10^{7} \mathrm{~V} / \mathrm{m}=10^{5} \mathrm{~V} / \mathrm{cm}$. Typical values for the field components in the above units are $10^{-3} \sim 10^{-2}$ for $\xi_{\perp}, 0.1 \sim 1$ for $\xi_{\|}$and $10^{-5} \sim 10^{-4}$ for $\epsilon$.

In the case of protons, the unit of length is the same as for electrons, but the unit of time must change so that the equations of motion are valid in the dimensionless form $(4,5,6)$. The unit of time becomes $1.04 \times 10^{-6} \mathrm{~s}$, yielding a new unit of velocity $5.44 \times 10^{5} \mathrm{~m} / \mathrm{s}$ and a new unit of energy $3.09 \times 10^{-3} \mathrm{MeV}$. The unit of the magnetic field is unaltered, but the unit of electric field becomes $5.44 \times 10^{3} \mathrm{~V} / \mathrm{m}$. Thus, the only changes when studying proton orbits are in the value of $\epsilon$ (typical values $\epsilon=$ $10^{-2} \sim 10^{-1}$ ) and in the timestep of the integration routine.

Integrating Eqs. (3), (5) once with respect to $t$ and substituting the result into (4) yields:

$\frac{\mathrm{d} x}{\mathrm{~d} t}=\xi_{\|} y-\xi_{\perp} z+c_{1}$

$\frac{\mathrm{d}^{2} y}{\mathrm{~d} t^{2}}+\xi_{\|}^{2} y=-\left(\epsilon t+\xi_{\perp} x+\frac{1}{2} y^{2}+c_{2}\right) y+\xi_{\|}\left(\xi_{\perp} z-c_{1}\right)$

$\frac{\mathrm{d} z}{\mathrm{~d} t}=\epsilon t+\xi_{\perp} x+\frac{1}{2} y^{2}+c_{2}$.

The constants $c_{1}$ and $c_{2}$, for zero initial velocities are given by:

$c_{1}=-\xi_{\|} y_{0}+\xi_{\perp} z_{0} \quad c_{2}=-\xi_{\perp} x_{0}-\frac{1}{2} y_{0}^{2}$

where $x_{0}, y_{0}, z_{0}$ are the initial coordinates of the particle.

The remaining part of the paper deals with the analysis of the orbits induced by Eqs. (3)-(5), or (6)-(8). It should by stressed that the equations of motion are autonomous because the topology of the fields described by Eqs. (1) has been assumed to remain stationary in time. In reality, the fields in reconnecting regions are not stationary, but their evolution timescales are in general larger than the typical dynamical times for particles' orbits within an RCS.

\section{Parametric resonance}

For the current sheet to act as an accelerator, it is necessary that a particle stays within the sheet for a time long enough so that a significant amount of its electric potential energy is converted to kinetic energy. Litvinenko \& Somov (1993) made an analysis of the range of parameter values for $\xi_{\|}$, as a function of $\xi_{\perp}$ and $\epsilon$, where the oscillations of a particle's orbit in the $y$-axis, i.e., perpendicularily to the sheet, are stable. This analysis is based on perturbative expansions. Namely, the orbital functions $x(t), y(t), z(t)$ are developed as

$x(t)=x_{0}+x_{1}(t), y(t)=y_{0}+y_{1}(t), \quad z(t)=z_{0}+z_{1}(t)$.

In Eq. (9), $x_{1}(t)$ and $z_{1}(t)$ are terms of size $O(\epsilon)$ representing a cycloid motion with initial conditions $\left(x_{0}, y_{0}, z_{0}\right)$. In other words, the particle oscillates in the $z$-axis, while the motion in the $x$-axis is the combination of a constant drift with velocity $\epsilon / \xi_{\perp}$ and of an oscillation. Litvinenko \& Somov (1993) 
treated $y_{1}(t)$ as containing terms of both first and second degree in $\epsilon$. One then finds the equation:

$\frac{\mathrm{d}^{2} y_{1}}{\mathrm{~d} t^{2}}+\left(\xi_{\|}^{2}+\epsilon \frac{\sin \left(\xi_{\perp} t\right)}{\xi_{\perp}}\right) y_{1}=\xi_{\|}^{2} y_{0}+\left(1-\cos \left(\xi_{\perp} t\right)\right) \epsilon \frac{\xi_{\|}}{\xi_{\perp}}$

By introducing a translation of the origin $Y=y_{1}-Y_{0}$, where $Y_{0}=y_{0}+\epsilon /\left(\xi_{\|} \xi_{\perp}\right)$, Eq. (10) takes the form:

$\frac{\mathrm{d}^{2} Y}{\mathrm{~d} t^{2}}+\xi_{\|}^{2}\left(1+\frac{\epsilon}{\xi_{\|}^{2} \xi_{\perp}} \sin \left(\xi_{\perp} t\right)\right) Y=$

$-\epsilon\left(y_{0}+\frac{\epsilon}{\xi_{\|} \xi_{\perp}}\right) \frac{\sin \left(\xi_{\perp} t\right)}{\xi_{\perp}}-\epsilon \cos \left(\xi_{\perp} t\right) \frac{\xi_{\|}}{\xi_{\perp}}$

The form of Eq. (11) is preferable because the right hand side is now a periodic function of time with period equal to $2 \pi / \xi_{\perp}$. Equation (11) is a particular case of a Mathieu equation (e.g. Ince 1926). Litvinenko \& Somov (1993) claimed that the condition

$\xi_{\|}^{2} \geq \frac{\epsilon}{\xi_{\perp}}$

is sufficient for the orbits to be stable, i.e., for $y(t)$ making stable oscillations. The argument is that if condition (12) is fulfilled, the unperturbed frequency appearing in the 1.h.s. of Eq. (11) is real-valued for all $t$. The physical meaning of the criterion provided by Eq. (12) is that if the longitudinal magnetic field $\xi_{\|}$is strong enough, the particles follow orbits almost parallel to the direction of the longitudinal field, ( $z$-axis), which is also parallel to the electric field. Thus the particles stay within the current sheet and they are being accelerated by the electric field.

However, the condition given by Eq. (12) is not sufficient to ensure stability of the orbits. A detailed study of the solutions of Eq. (11) can be obtained by the theory of Mathieu equations (Ince 1926). In particular, Eqs. (10) and (11) are special examples of dynamical systems where the so-called parametric resonance phenomenon (e.g. Arnold 1978) is manifested. One finds that there is a periodic solution of Eq. (11), namely a solution of the form $Y(t+T)=Y(t)$ where $T=2 \pi / \xi_{\perp}$. We determine this orbit numerically, by considering a Poincaré surface of section at times $t=n T$, with $n$ integer, for the solutions of Eq. (11). The successive iterates of the orbit on the surface of section define a two-dimensional area-preserving mapping

$Y^{\prime}=f\left(Y, P_{Y}\right), \quad P_{Y}^{\prime}=g\left(Y, P_{Y}\right)$

where $Y=Y(t), P_{Y}=\dot{Y}(t)$ are the values corresponding to any solution of (11), and $Y^{\prime}=Y(t+T), P_{Y}^{\prime}=\dot{Y}(t+T)$. $Y(t+T), P_{Y}(t+T)$ are uniquely determined as the solutions of the same differential equation at time $t^{\prime}=t+T$, with initial conditions $Y(t)$ and $P_{Y}(t)$ at time $t$. Thus, in (13), $Y^{\prime}$ and $P_{Y}^{\prime}$ are written simply as functions of $Y$ and $P_{Y}$. A periodic orbit corresponds to a fixed point of the mapping (13), namely to a pair of values $Y_{0}, P_{Y 0}$ such that $Y^{\prime}=Y_{0}, P_{Y}^{\prime}=P_{Y 0}$. This point is determined by finding numerically, with a Newton-Raphson scheme, the root of the system of equations

$$
F\left(Y, P_{Y}\right)=Y-f\left(Y, P_{Y}\right)=0, \quad G\left(Y, P_{Y}\right)=P_{Y}-g\left(Y, P_{Y}\right)=0 .
$$

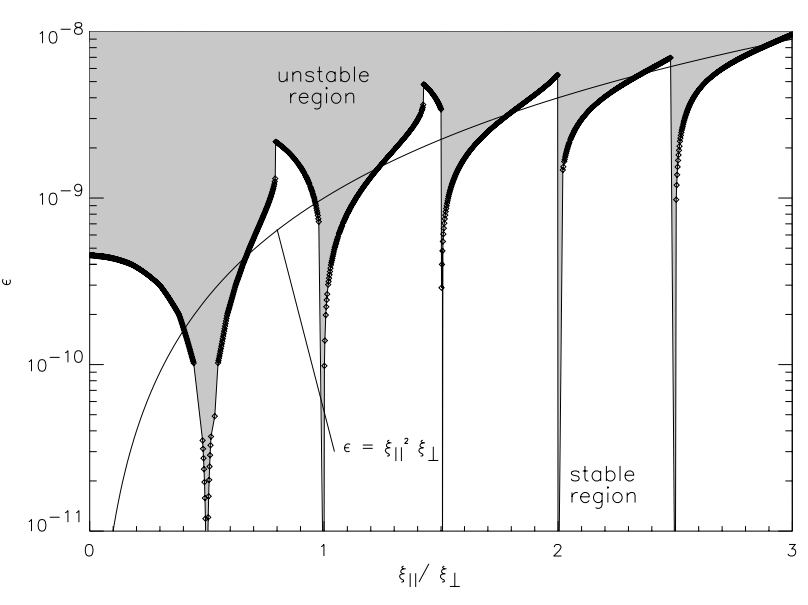

Fig. 2. Parametric resonance in the equations of motion (Eq. (11)). The dotted curve indicates the transition values of the parameters $\epsilon$ and $\xi_{\|} / \xi_{\perp}$ for which the orbits turn from stable to unstable. The criterion (12) is superposed (curve without dots).

The linear stability of the periodic solution is determined by the eigenvalues of the monodromy matrix $A$ which is the Jacobian of the mapping (13) calculated at the fixed point $\left(Y_{0}, P_{Y 0}\right)$. According to the theory of parametric resonance, as $\xi_{\|}$increases, the periodic orbit $\left(Y_{0}, P_{Y 0}\right)$ undergoes an infinity of transitions from stable to unstable. For $\epsilon$ small, these transitions occur near the values where the angular frequency $\omega_{0}$ of the unperturbed oscillator (for $\epsilon=0$ ) becomes a multiple of $1 / 2$, namely $\omega_{0}=n / 2$. In the case of the mapping (13) defined by Eq. (11), for fixed $\xi_{\perp}, \omega_{0}$ is proportional to $\xi_{\|}$. Thus, an infinity of transitions from stability to instability are expected as $\xi_{\|}$ increases. These transitions are shown in Fig. 2. The dotted line indicates the critical parameter values where the periodic orbit turns from stable to unstable. The dotted curve has branches which meet in pairs at specific points on the $x$-axis, corresponing to the parameter value $\epsilon=0$. As shown in Fig. 2, these points are precisely the multiples of $1 / 2$.

The domains of instability defined for low multiples of $1 / 2$ are much larger than those defined for high multiples of $1 / 2$. Thus, instability zones of considerable width exist for relatively low values of $\xi_{\|}$. For super-Dreicer electric fields, these zones are very narrow so that the criterion (12) is an acceptable approximation (solid line in Fig. 2). Nevertheless, for any value of $\epsilon>0$, instability domains, of narrower and narrower width, exist for arbitrarily large values of $\xi_{\|}$.

\section{Hamiltonian reduction and theoretical study of the orbits}

\subsection{Hamiltonian reduction}

The results of the previous section were based on a perturbative treatment of the equations of motion (3)-(5). In this section we examine the full form of these equations of motion. These can be derived from a three degrees of freedom autonomous Hamiltonian function

$H=\frac{1}{2}\left(p_{x}+\xi_{\|} y\right)^{2}+\frac{1}{2} p_{y}^{2}+\frac{1}{2}\left(p_{z}+\xi_{\perp} x+\frac{1}{2} y^{2}\right)^{2}-\epsilon z$ 
where the canonical momenta $p_{x}, p_{y}, p_{z}$ are given by the sum of the velocity and of the vector potential components

$p_{x}=\dot{x}-\xi_{\|} y, \quad p_{y}=\dot{y}, \quad p_{z}=\dot{z}-\xi_{\perp} x-\frac{1}{2} y^{2}$.

One verifies immediately that Hamilton's equations

$\dot{x}=\frac{\partial H}{\partial p_{x}}, \quad \dot{p_{x}}=-\frac{\partial H}{\partial x}$

(and similarly for $y, p_{y}$ and $z, p_{z}$ ), lead to the equations of motion (3)-(5).

Equations (17) admit an exact second integral of motion independent of and in involution with the Hamiltonian (15):

$I_{2}=p_{x}+\xi_{\perp} z$

We find $\dot{I}_{2}=\left\{I_{2}, H\right\}=0$, where $\{\cdot, \cdot\}$ stands for the Poisson bracket operator. Despite the existence of this second integral, the 3DOF Hamiltonian system (15) is not integrable. One more integral, independent of and in involution with both $H$ and $I_{2}$, is required to ensure integrability of the system (15). Based on some heuristic considerations, Litvinenko (1993) proposed that there should be an approximate third integral of the motion which renders regular the character of the orbits within the domain of its validity. Indeed, such an approximate integral is found in Sect. 4.3 below.

The existence of a second integral $I_{2}$ allows one to reduce the system, for any constant value of $I_{2}$, to a two degrees of freedom Hamiltonian system. In general the algorithm for this reduction (Arnold \& Novikov 1995) leads to implicit formulae. However, in the present case the reduction can be carried out explicitly. Indeed if one uses the pairs of variables $\left(y, p_{y}\right)$ and $\left(z, c_{4}\right)$, where $c_{4}=\xi_{\perp} x+p_{z}$, then the use of the original equations of motion and of the expression (18) for $I_{2}$ yields the equations

$$
\begin{aligned}
\dot{y} & =p_{y} \\
\dot{z} & =c_{4}+\frac{1}{2} y^{2} \\
\dot{p}_{y} & =-y\left(c_{4}+\frac{1}{2} y^{2}\right)-\xi_{\|}\left(I_{2}-\xi_{\perp} z+\xi_{\|} y\right) \\
\dot{c}_{4} & =\xi_{\perp}\left(I_{2}-\xi_{\perp} z+\xi_{\|} y\right)+\epsilon
\end{aligned}
$$

which are, precisely, Hamilton's equations deduced from the 2DOF Hamiltonian:

$H=\frac{1}{2} p_{y}^{2}+\frac{1}{2}\left(c_{4}+\frac{1}{2} y^{2}\right)^{2}+\frac{1}{2}\left(I_{2}-\xi_{\perp} z+\xi_{\|} y\right)^{2}-\epsilon z$.

The reduction of the original system to a Hamiltonian system of two degrees of freedom is a quite helpful step, because many well known analytical and numerical tools of study are more effectively applicable in two than in three degrees of freedom Hamiltonian systems. In particular, the theory of normal forms can be used to extract analytically the main characteristics of the orbits, and the method of the Poincare surface of section can be used to identify the regular or chaotic character of particular orbits.

\subsection{Equilibrium points when $\epsilon=0$}

For typical values of the electric and magnetic fields in solar RCS, the parameter $\epsilon$ is small, $\epsilon \ll 1$, i.e., the term $\epsilon z$ in the Hamiltonian (20) can be considered as a perturbation term with respect to the Hamiltonian $H_{0}$ without electric field, namely

$$
H_{0}=\frac{1}{2} p_{y}^{2}+\frac{1}{2}\left(c_{4}+\frac{1}{2} y^{2}\right)^{2}+\frac{1}{2}\left(I_{2}-\xi_{\perp} z+\xi_{\|} y\right)^{2}
$$

The Hamiltonian (21) posesses equilibrium points that can serve as starting points for a theoretical analysis of the orbits. Hamilton's equations of motion for (21) are

$$
\begin{aligned}
\dot{y} & =p_{y} \\
\dot{z} & =c_{4}+\frac{1}{2} y^{2} \\
\dot{p}_{y} & =-y\left(c_{4}+\frac{1}{2} y^{2}\right)-\xi_{\|}\left(I_{2}-\xi_{\perp} z+\xi_{\|} y\right) \\
\dot{c}_{4} & =\xi_{\perp}\left(I_{2}-\xi_{\perp} z+\xi_{\|} y\right) .
\end{aligned}
$$

For any value of the integral $I_{2}$, the family of points

$p_{y 0}=0, \quad z_{0}=\frac{1}{\xi_{\perp}}\left(\xi_{\|} y_{0}+I_{2}\right), \quad c_{40}=-\frac{1}{2} y_{0}^{2}$,

for $y_{0}$ arbitrary, are equilibrium points of the equations of motion (22). The stability of the whole family is obtained by linearizing Eqs. (22). Taking $y=y_{0}+Y, z=z_{0}+Z, p_{y}=p_{y 0}+P_{Y}$, $c_{4}=c_{40}+P_{Z}$, where $Y, P_{Y}, Z$ and $P_{Z}$ are small quantities, yields the variational equations of motion

$$
\begin{aligned}
\dot{Y} & =P_{Y} \\
\dot{Z} & =P_{Z}+y_{0} Y \\
\dot{P}_{Y} & =-y_{0} P_{Z}-\left(y_{0}^{2}+\xi_{\|}^{2}\right) Y+\xi_{\|} \xi_{\perp} Z \\
\dot{P}_{Z} & =\xi_{\perp} \xi_{\|} Y-\xi_{\perp}^{2} Z .
\end{aligned}
$$

Differentiation of Eq. (24) with respect to time yields a linear system of the form

$\ddot{Q}=A Q$

where $Q \equiv\left(Y, Z, P_{Y}, P_{Z}\right)$ and $A$ is a $4 \times 4$ matrix with constant coefficients. The equation

$\operatorname{det}\left(A+\omega^{2} I\right)=0$

gives the eigenvalues (characteristic frequencies) which determine the stability of the equilibrium point. We find

$\omega_{1}^{2}=\xi_{\|}^{2}+\xi_{\perp}^{2}+y_{0}^{2}, \quad \omega_{2}^{2}=0$.

Thus, the whole family of equilibria are marginally simply unstable. This is nothing but the fact that, in the linear approximation, the motion around any equilibrium consists of a drift along the magnetic field lines and an oscillation (projection of a gyration) in the transverse plane to the field lines. However, as shown in the sequel, the presence of nonlinear terms in the equations of motion results in a second small "mirror" frequency being introduced along the field lines. 


\subsection{Normal form}

Without loss of generality, we consider in detail the case $I_{2}=0$, and the equilibrium point of (21) $y_{0}=z_{0}=p_{y 0}=c_{40}=0$. A linear canonical transformation diagonalizes the quadratic part of the Hamiltonian (20). By introducing new canonical variables

$u_{1}=\frac{-\xi_{\|} y+\xi_{\perp} z}{\sqrt{\xi_{\|}^{2}+\xi_{\perp}^{2}}}, \quad p_{1}=\frac{-\xi_{\|} p_{y}+\xi_{\perp} c_{4}}{\sqrt{\xi_{\|}^{2}+\xi_{\perp}^{2}}}$
$u_{2}=\frac{\xi_{\perp} y+\xi_{\|} z}{\sqrt{\xi_{\|}^{2}+\xi_{\perp}^{2}}}, \quad p_{2}=\frac{\xi_{\perp} p_{y}+\xi_{\|} c_{4}}{\sqrt{\xi_{\|}^{2}+\xi_{\perp}^{2}}}$

the Hamiltonian (21) takes the form

$H=H_{2}+H_{3}+H_{4}$

where

$$
\begin{aligned}
H_{2}= & \frac{1}{2} p_{1}^{2}+\frac{1}{2}\left(\xi_{\|}^{2}+\xi_{\perp}^{2}\right) u_{1}^{2}+\frac{1}{2} p_{2}^{2} \\
H_{3}= & \frac{1}{2\left(\xi_{\|}^{2}+\xi_{\perp}^{2}\right)^{3 / 2}}\left(\xi_{\|} \xi_{\perp}^{2} u_{2}^{2} p_{2}+\xi_{\perp}^{3} u_{2}^{2} p_{1}+\xi_{\|}^{3} u_{1}^{2} p_{2}\right. \\
& \left.\quad+\xi_{\|}^{2} \xi_{\perp} u_{1}^{2} p_{1}-2 \xi_{\|}^{2} \xi_{\perp} u_{1} u_{2} p_{2}-2 \xi_{\|} \xi_{\perp}^{2} u_{1} u_{2} p_{1}\right) \\
H_{4}= & \frac{1}{8\left(\xi_{\|}^{2}+\xi_{\perp}^{2}\right)^{2}}\left(\xi_{\perp}^{4} u_{2}^{4}-4 \xi_{\perp}^{3} \xi_{\|} u_{1} u_{2}^{3}\right. \\
& \left.+6 \xi_{\perp}^{2} \xi_{\|}^{2} u_{1}^{2} u_{2}^{2}-4 \xi_{\perp} \xi_{\|}^{3} u_{1}^{3} u_{2}+\xi_{\|}^{4} u_{1}^{4}\right)
\end{aligned}
$$

The terms $H_{3}$ and $H_{4}$ can be considered as perturbation terms with respect to the quadratic term $H_{2}$. To study the effect of the nonlinear terms $\mathrm{H}_{3}$ and $H_{4}$, we find a "normalizing transformation" and associated normal form for the Hamiltonian (29). A normalizing transformation is a near-identity canonical transformation to new variables $u_{i}^{\prime}=u_{i}+\ldots, p_{i}^{\prime}=p_{i}+\ldots$ such that the Hamiltonian (29), expressed in the new variables $\left(u_{i}^{\prime}, p_{i}^{\prime}\right)$ takes a simpler form (the normal form) which renders transparent the nature of the solutions to Hamilton's equations, i.e., the nature of the orbits. The general theory of normal forms for Hamiltonian systems, developed by Poincaré (1892) and Birkhoff (1927), does not cover the case when one of the frequencies is equal to zero, as in the case of the Hamiltonian $H_{2}$. The appropriate theory was developed by Contopoulos \& Vlahos (1975), using the Birkhoff - von Zeipel method, and by Dragt \& Finn (1979) and Engel et al. (1995), using the method of canonical transformations via the composition of Lie series. Here we implement a simplified version of the algorithm of Dragt \& Finn (1979). Details of this algorithm are given in the Appendix.

Introducing action-angle variables $\left(J_{1}, \phi_{1}\right)$ given by:

$u_{1}=\sqrt{\frac{2 J_{1}}{\omega_{10}}} \cos 2 \phi_{1}, \quad p_{1}=\sqrt{2 \omega_{10} J_{1}} \sin 2 \phi_{1}$

where $\omega_{10}=\sqrt{\xi_{\perp}^{2}+\xi_{\|}^{2}}$, the quadratic part of the Hamiltonian is given by

$H_{2}=\omega_{10} J_{1}+\frac{p_{2}^{2}}{2}$.

In the absence of $H_{3}$ and $H_{4}$, the action $J_{1}$ is an integral of motion (adiabatic invariant). However, the terms $\mathrm{H}_{3}$ and
$H_{4}$ depend on the angle $\phi_{1}$, so that $J_{1}$ is no longer an integral of the motion. We thus introduce new canonical variables $J_{1}^{\prime}, \phi_{1}^{\prime}, u_{2}^{\prime}, p_{2}^{\prime}$ by a near identity transformation so that the angle $\phi_{1}^{\prime}$ is eliminated in the transformed Hamiltonian (normal form). The normal form $Z \equiv H\left(J_{1}^{\prime}, \phi_{1}^{\prime}, u_{2}^{\prime}, p_{2}^{\prime}\right)$ contains only terms which are even in the canonical variables. Up to terms of sixth degree, it is given by:

$Z=Z_{2}+Z_{4}+Z_{6}+\ldots$

where

$Z_{2}=\omega_{10} J_{1}^{\prime}+\frac{p_{2}^{\prime 2}}{2}$

$Z_{4}=\frac{\xi_{\|}^{2} J_{1}^{\prime 2}}{16 \omega_{10}^{4}}+\frac{\xi_{\perp}^{2} J_{1}^{\prime} u_{2}^{\prime 2}}{2 \omega_{10}^{3}}$

$Z_{6}=\frac{\xi_{\|}^{2} J_{1}^{\prime 3}}{\omega_{10}^{9}}\left(\frac{5 \xi_{\perp}^{2}}{576}-\frac{\xi_{\|}^{2}}{256}\right)+\frac{\xi_{\perp}^{2} J_{1}^{\prime 2} u_{2}^{\prime 2}}{16 \omega_{10}^{8}}\left(25 \xi_{\perp}^{2}-8 \xi_{\|}^{2}\right)-\frac{7 \xi_{\perp}^{4} J_{1}^{\prime} u_{2}^{\prime 4}}{24 \omega_{10}^{7}}$.

The new variables are given in terms of the old variables by the relations (up to fourth degree):

$J_{1}^{\prime}=J_{1}+\frac{\xi_{\|}^{2} \xi_{\perp}^{2}\left(2 \xi_{\perp}^{2}-\xi_{\|}^{2}\right)}{16\left(\xi_{\|}^{2}+\xi_{\perp}^{2}\right)^{9 / 2}} J_{1}^{2}+F_{1}\left(J_{1}, \phi_{1}, u_{2}, p_{2}\right)$

$u_{2}^{\prime}=u_{2}+U_{2}\left(J_{1}, \phi_{1}, u_{2}, p_{2}\right)$,

$p_{2}^{\prime}=p_{2}+\frac{\xi_{\|}^{3} J_{1}}{2\left(\xi_{\|}^{2}+\xi_{\perp}^{2}\right)^{2}}+P_{2}\left(J_{1}, \phi_{1}, u_{2}, p_{2}\right)$

where the functions $F_{1}, U_{2}$ and $P_{2}$ are trigonometric functions with respect to the angle $\phi_{1}\left(F_{1}, U_{2}\right.$ are $O\left(J_{1}^{3 / 2}\right)$ while $P_{2}$ is $\left.O\left(J_{1}\right)\right)$. Thus, the values of the new variables along a particular orbit fluctuate in general around the values of the respective old variables along the same orbit. Furthermore, the values of $J_{1}^{\prime}$ are shifted with respect to the values of $J_{1}$ by a second order correction $O\left(J_{1}^{2}\right)$. This is expected by the general normal form theory. However, the values of $p_{2}^{\prime}$ are shifted with respect to the values of $p_{2}$ by a first order correction $O\left(J_{1}\right)$. This phenomenon appears only in the particular case of the normal form considered here.

Since the angle $\phi_{1}^{\prime}$ does not appear in $Z, J_{1}^{\prime}$ becomes a "formal integral" of the motion. This integral is approximate because the series expressions are truncated at order six. However, even at this order of truncation the normal form allows one to extract the main characteristics of the orbits with a quite high accuracy.

By a rearrangement of terms $Z$ takes the form:

$Z=\omega_{10} J_{1}^{\prime}+\frac{1}{2}\left(p_{2}^{\prime 2}+\omega_{2}^{2}\left(J_{1}^{\prime}\right) u_{2}^{\prime 2}\right)+b\left(J_{1}^{\prime}\right)-c\left(J_{1}^{\prime}\right) u_{2}^{\prime 4}+\ldots$

where

$\omega_{2}^{2}=\frac{\xi_{\perp}^{2} J_{1}^{\prime}}{\omega_{10}^{3}}+\frac{\xi_{\perp}^{2} J_{1}^{\prime 2}}{8 \omega_{10}^{8}}\left(25 \xi_{\perp}^{2}-8 \xi_{\|}^{2}\right)+\ldots$

$b\left(J_{1}^{\prime}\right)=\frac{\xi_{\|}^{2} J_{1}^{\prime 2}}{16 \omega_{10}^{4}}+\frac{\xi_{\|}^{2} J_{1}^{\prime 3}}{\omega_{10}^{9}}\left(\frac{5 \xi_{\perp}^{2}}{576}-\frac{\xi_{\|}^{2}}{256}\right)+\ldots$

and

$c\left(J_{1}^{\prime}\right)=\frac{7 \xi_{\perp}^{4} J_{1}^{\prime}}{24 \omega_{10}^{7}}+\ldots$ 


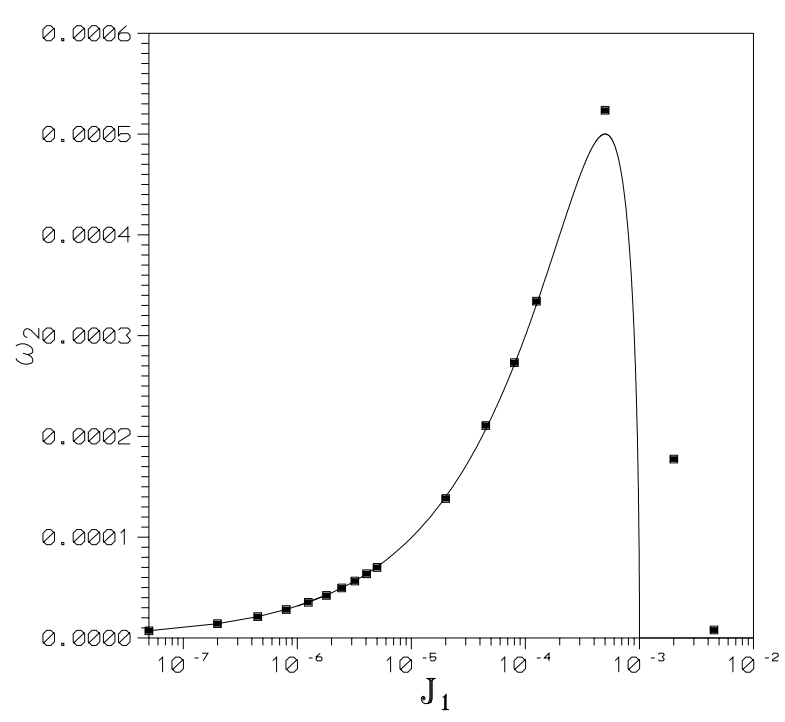

Fig. 3. Comparison between the numerically calculated frequency $\omega_{2}$, as a function of $J_{1}$, and the theoretical prediction by the normal form analysis (Eq. (34)).

Considering any constant value of the adiabatic invariant $J_{1}^{\prime}=$ const., the Hamiltonian $Z$ may be viewed as an one degree of freedom Hamiltonian for the canonical variables $\left(u_{2}^{\prime}, p_{2}^{\prime}\right)$. If $\omega_{2}^{2}\left(J_{1}^{\prime}\right)>0$, the motion in the second degree of freedom is a harmonic oscillation with frequency equal to $\omega_{2}$ plus corrections due to higher order terms in $u_{2}^{\prime}$. This oscillation causes a "magnetic mirror" effect. The condition $\omega_{2}^{2}\left(J_{1}^{\prime}\right)>0$ is fulfilled for
$0<J_{1}^{\prime}<\infty$
if $\xi_{\|}^{2}<25 \xi_{\perp}^{2} / 8$
$0<J_{1}^{\prime}<\frac{8 \omega_{10}^{5}}{8 \xi_{\|}^{2}-25 \xi_{\perp}^{2}}$
if $\xi_{\|}^{2}>25 \xi_{\perp}^{2} / 8$

At the limit $\xi_{\|} \gg \xi_{\perp}$, the transformation (28) tends to the transformation

$u_{1} \simeq-y, \quad u_{2} \simeq z$.

Thus, when $\xi_{\|} \gg \xi_{\perp}$ the slow "mirror" oscillation is practically aligned with the direction of the longitudinal field, while there is a fast gyration, with frequency of the order of $\xi_{\|}$transversally to the longitudinal field. This limit corresponds to the "guiding center approximation". The frequency of the mirror oscillation

$\omega_{2}=\sqrt{\frac{\xi_{\perp}^{2} J_{1}^{\prime}}{\omega_{10}^{3}}+\frac{\xi_{\perp}^{2} J_{1}^{\prime 2}}{8 \omega_{10}^{8}}\left(25 \xi_{\perp}^{2}-8 \xi_{\|}^{2}\right)}$

initially increases as $J_{1}^{\prime}$ increases, while it reaches a maximum at the value $J_{1}^{\prime}=4 \omega_{10}^{5} /\left(8 \xi_{\|}^{2}-25 \xi_{\perp}^{2}\right)$. These results are confirmed by a comparison of Eq. (37) with a numerical calculation of the mirror frequency along various orbits for different values of $J_{1}^{\prime}$ (Fig. 3).

\subsection{Poincaré surfaces of section. Regular and chaotic orbits}

All the orbits of energy $E$ which intersect a particular plane $y=$ const. with $\dot{y}>0$ (or $\dot{y}<0$ ) determine a Poincaré surface of section $\left(z, c_{4}\right)$. Such sections are quite useful in visualizing the dynamics of the orbits. In particular, regular orbits lie on invariant curves in the Poincaré section, while chaotic orbits fill stochastic domains in the same section.

Figure 4 shows an example of Poincaré surfaces of section for the Hamiltonian (21). The section is the plane $\left(z, c_{4}\right)$, for $y=0, \dot{y}>0$. For $y=0$ we have $c_{4}=\dot{z}$ (see Eq. (22)). Thus, the sections shown below will be referred to as $(z, \dot{z})$. In fact, the constant value of the second integral $I_{2}=\dot{x}+\xi_{\perp} z-\xi_{\|} y=0$ yields, on the Poincaré section $y=0$, the relation $\dot{x}=-\xi_{\perp} z$. Thus, the section plane $(z, \dot{z})$ of the 2DOF Hamiltonian (21) is equivalent to the plane $(\dot{x}, \dot{z})$ of the original 3DOF Hamiltonian (15), with $\epsilon=0$. This means that all the structures observed in the above Poincaré surface of section can be translated in terms of the three velocity components $(\dot{x}, \dot{y}, \dot{z})(\dot{y}$ is obtained by the constant energy condition). This reflects the fact that the equations of motion are invariant under translations in the direction of the $z$ axis. In fact, a translation $z \rightarrow z+z_{0}$ is equivalent to a different value of $I_{2}$, i.e., $I_{2} \rightarrow I_{2}+\xi_{\perp} z_{0}$.

In all the sections shown in Fig. 4, the section points are limited in a domain defined by a limiting outermost closed curve. This is the curve of zero velocity on the Poincaré section, corresponding to orbits that reach the section plane tangently. In fact, for any fixed value of the energy $H=E$, and for $y=0$, the velocity $\dot{y}=p_{y}$ can be determined from the Hamiltonian (21)

$\dot{y}=p_{y}= \pm \sqrt{2 E-\dot{z}^{2}-\xi_{\perp}^{2} z^{2}}$.

The quantity under the square root must be positive. The limiting curve on the surface of section is then given by

$\dot{z}^{2}+\xi_{\perp}^{2} z^{2}=2 E$

i.e., it is an ellipse centered at $(z, \dot{z})=(0,0)$, with axial ratio equal to $\xi_{\perp}$.

In Fig. 4a the energy is $E=10^{-4}$ and the parameters are $\xi_{\perp}=0.001$ and $\xi_{\|}=0.1$. In the surface of section of Fig. 4a most orbits are regular, i.e., they lie on invariant curves. The 'formal integral' approximation is valid for such orbits. However, as the effective perturbation $\xi_{\perp} / \xi_{\|}$increases, or as the energy $E$ increases for fixed $\xi_{\perp} / \xi_{\|}$, many tori are destroyed, and chaos is introduced in the system. These phenomena are shown in Figs. 4b,c,d. Figures 4b,c show the surface of section for the same energy and $\xi_{\|}$, but for $\xi_{\perp}=0.01$ and 0.02 respectively. The size of domain of chaotic motion increases in general as $\xi_{\perp} / \xi_{\|}$increases. In particular, as $\xi_{\perp}$ increases, the chaos proceeds mainly from an outer domain inwards, towards a central periodic orbit (marked by $\mathrm{O}$ ). For $\xi_{\perp}$ sufficiently large, the regular domain is defined by an outermost invariant curve which is a closed curve around $\mathrm{O}$ (Fig. 4c).

On the other hand, the intersections of a chaotic orbit with the upper part of the surface of section give the impression of forming arcs that are similar to segments of open invariant curves as those of Fig. 4a. However, the same orbit, after making a number of intersections along one arc, goes away from the surface of section and later returns to it giving points on a different arc. Thus, the orbit does not belong to an invariant curve but it is a chaotic orbit which behaves almost regularly for some transient time intervals. This phenomenon is 

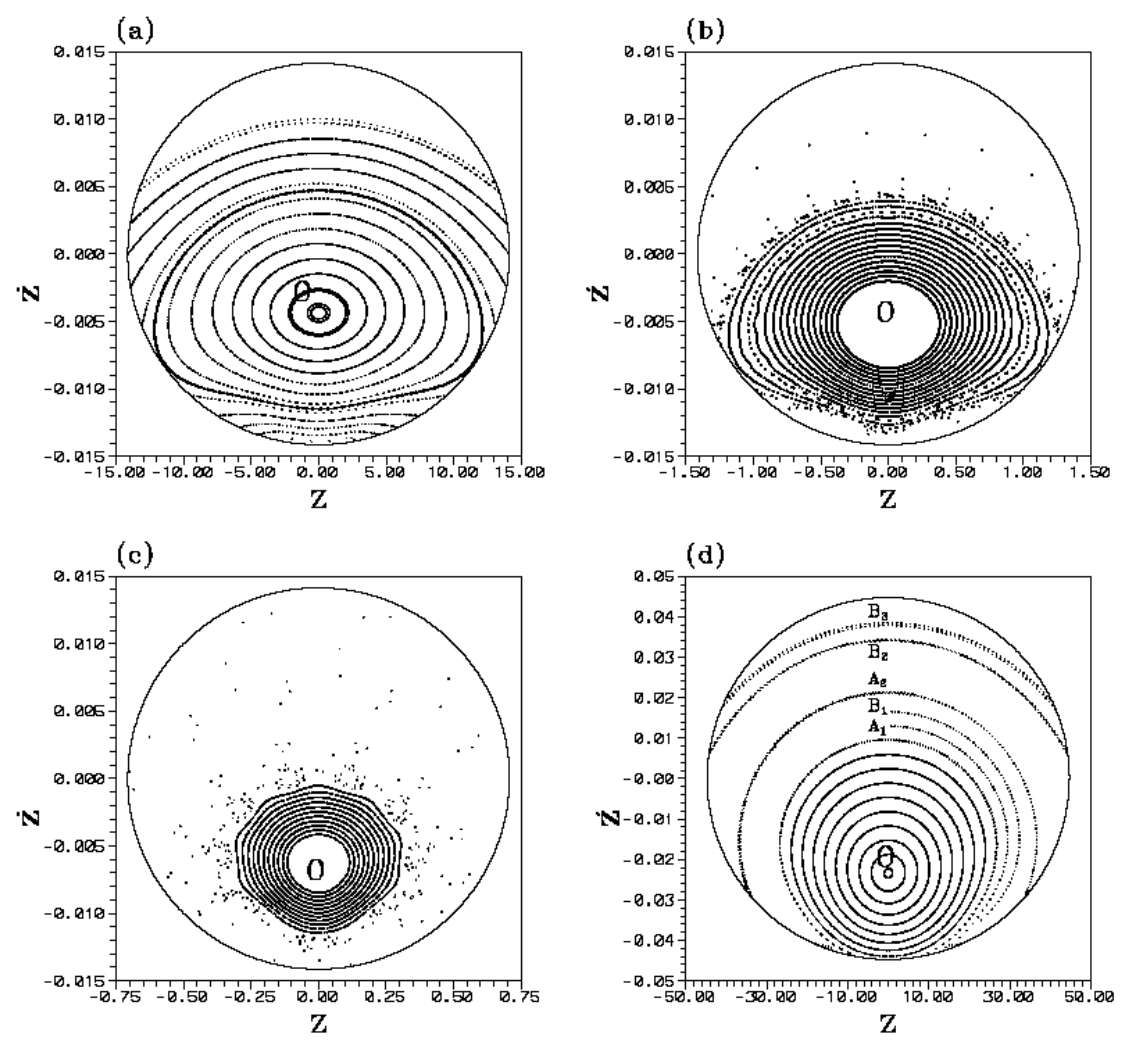

Fig. 4. a) Poincaré surfaces of section $(z, \dot{z}), y=0, \dot{y}>0$, for the Hamiltonian (21), for $\xi_{\|}=0.1$ and a) $\left.\xi_{\perp}=0.001, E=10^{-4} \mathbf{b}\right) \xi_{\perp}=0.01$, $E=10^{-4}$ c) $\xi_{\perp}=0.02, E=10^{-4}$ d) $\xi_{\perp}=0.001, E=10^{-3}$.

clearly seen on the Poincaré surface of section as the energy $E$ increases. Figure 4d shows the surface of section for the same parameters as in Fig. 4a, but for the energy $E=10^{-3}$. At this energy many orbits around $O$ are still regular. However, the orbits with initial conditions $(z, \dot{z})=(0,0.012)$ (point A1) and $(z, \dot{z})=(0,0.016)$ (point B1) are chaotic. In particular, the orbit with the initial condition A1 forms an inner arc down to a point intersecting the limiting curve, but the next consequent is on the arc A2, which is further away. Similarly, the orbit starting at $\mathrm{B} 1$ gives intesections forming an arc which intersects the limiting curve, but the consecutive returns of the same orbit to the surface of section are on the arc B2, and later on B3. Thus these orbits do not lie on invariant curves, but they are chaotic. Similar arcs exist in Fig. 4b, but they are less visible.

The phenomenon of appearance of arcs that are consecutively intersected by weakly chaotic orbits is well known in this type of system (e.g. Büchner \& Zelenyi 1989), but also in other types of systems such as rotating galactic potentials (see Contopoulos 2002). In particular, the consecutive crossings can be described in terms of a mapping similar to the Chririkov standard map, i.e., this phenomenon is similar to Fermi acceleration (Lichtenberg \& Lieberman 1992).

The fixed point $O$ corresponds to the equilibrium point $u_{2}^{\prime}=p_{2}^{\prime}=0$ of the Hamiltonian (34) written in normal form. Now, recalling that $p_{2}^{\prime}$ contains a first order correction $O(J)$ with respect to $p_{2}$, given by Eq. (33), the equilibrium point $\left(u_{2}^{\prime}, p_{2}^{\prime}\right)$ corresponds to the point

$\left(u_{2}, p_{2}\right) \simeq\left(0,-\frac{\xi_{\|}^{3} J_{1}}{2\left(\xi_{\|}^{2}+\xi_{\perp}^{2}\right)^{2}}\right)$.
Recalling the transformations (28), for $\xi_{\|} \gg \xi_{\perp}$, yields the approximate position of the fixed point $O$ in the old variables

$z=0, \quad \dot{z} \simeq-\frac{J_{1}}{2 \xi_{\|}}$

If $y_{0}$ is the amplitude of oscillations in the $y$-axis, in the neighborhood of the fixed point $O$, then, the action $J_{1}$, in the lowest approximation, is given by $J_{1}=\omega_{10} y_{0}^{2} / 2 \simeq \xi_{\|} y_{0}^{2} / 2$, which yields the position of the fixed point as

$z=0, \quad \dot{z} \simeq-\frac{y_{0}^{2}}{4}$

The invariant curves close to the point $O$ are deformed ellipses, i.e. they are closed curves surrounding the point $O$. However, at larger distances from $O$ the invariant curves are open. This effect is explained in Sect. 4.5 below.

Let us now give a non-zero value to the electric field $\epsilon$ in the Hamiltonian (20). The limiting surfaces of motion, for any value of the energy $E$, are given by setting $\dot{z}=c_{4}+y^{2} / 2=0$ and $\dot{y}=p_{y}=0$ in the Hamiltonian (20), yielding

$\xi_{\|}^{2} y^{2}+\xi_{\perp}^{2} z^{2}-2 \xi_{\|} \xi_{\perp} y z-2 \epsilon z=2 E$

which is a parabola on the plane $(y, z)$ open at the limit $y \rightarrow$ $\infty$. Thus, the limiting surface is open at the half-plane $y>0$ (signs are reversed for charges of opposite sign). This means that escapes from the plane $y=1$ are possible for any value of the energy, while escapes from the plane $y=-1$ are possible only if the minimum of the parabola is below $y=-1$. 
(a)

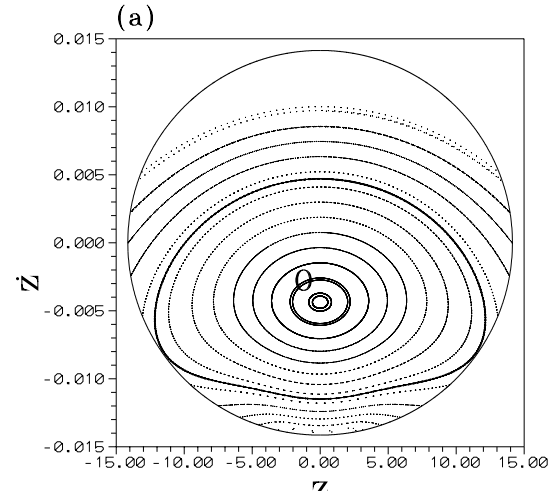

$\mathrm{Z}$

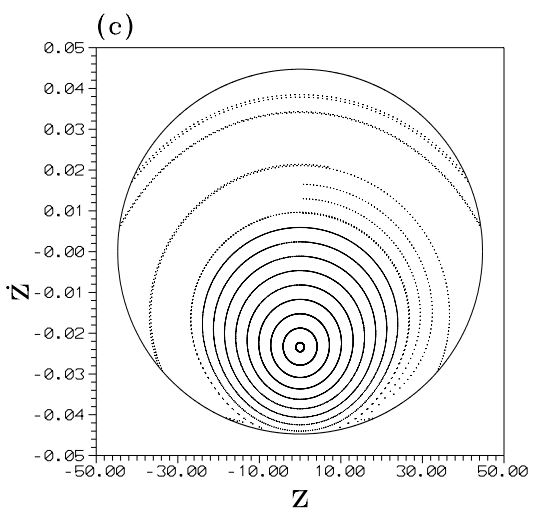

(b)

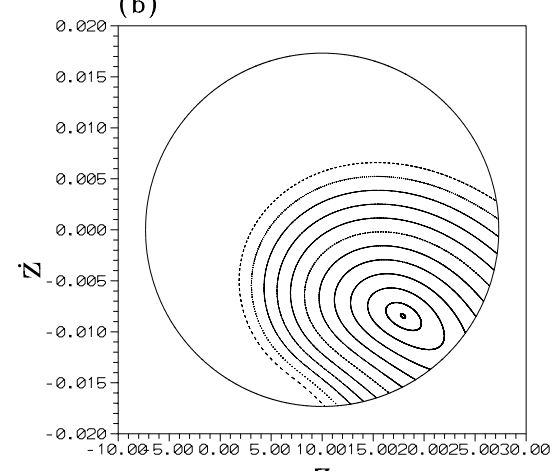

$\mathrm{Z}$

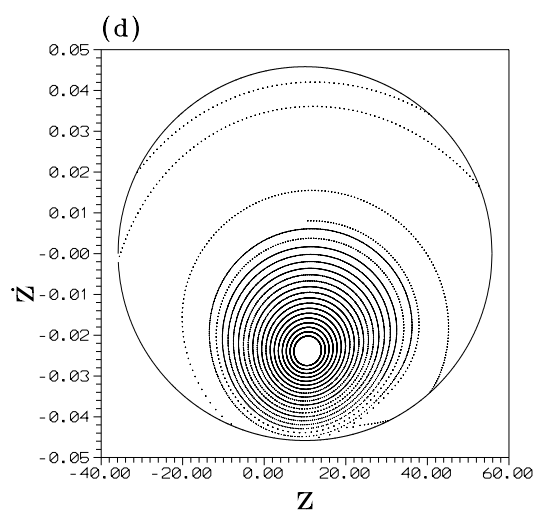

Fig. 5. Poincaré surfaces of section $(z, \dot{z}), y=0, \dot{y}>0$, for the Hamiltonian (20), with $\xi_{\|}=0.1, \xi_{\perp}=0.001$, and a) $\epsilon=0, E=10^{-4}$, b) $\epsilon=10^{-5}$, $E=10^{-4}$, c) $\epsilon=0, E=10^{-3}$, d) $\epsilon=10^{-5}, E=10^{-3}$.

If we consider any surface of section $(z, \dot{z})$ for $y=y_{s}, \dot{y}>0$, escapes are possible perpendicularly to the section plane. The limiting curve in such a section is given by

$\dot{z}^{2}+\xi_{\perp}^{2}\left(z-\frac{\xi_{\|} \xi_{\perp} y_{s}+\epsilon}{\xi_{\perp}^{2}}\right)^{2}=2 E+\frac{\epsilon}{\xi_{\perp}}\left(2 \xi_{\|} y_{s}+\frac{\epsilon}{\xi_{\perp}}\right)$,

Eq. (43) defines an ellipse which reduces to a point when

$y_{s, \min }=-\frac{\epsilon}{2 \xi_{\|} \xi_{\perp}}-\frac{E \xi_{\perp}}{\epsilon \xi_{\|}}$.

All the orbits reaching this point are bounced in the positive $y$ direction.

When $y_{s}=0$, the limiting curve reduces to

$\dot{z}^{2}+\xi_{\perp}^{2}\left(z-\frac{\epsilon}{\xi_{\perp}^{2}}\right)^{2}=2 E+\frac{\epsilon^{2}}{\xi_{\perp}^{2}}$.

Thus, the center of the limiting curve is displaced, with respect to the center of the limiting curve of the case $\epsilon=0$, by the quantity $\epsilon / \xi_{\perp}^{2}$. Equivalently, since $I_{2}=0$, this displacement corresponds to an increase of the average velocity $\dot{x}$ by $\epsilon / \xi_{\perp}$ for all the orbits with initial conditions in the section. This is nothing but the constant drift introduced by the electric field perpendicularly to $\epsilon$ and $\xi_{\perp}$.

Now, a numerical integration of the orbits shows that many regular orbits survive for $\epsilon \neq 0$, yielding invariant curves on the Poincare section which are displaced and deformed with respect to the invariant curves in the case $\epsilon=0$. Figure 5 shows the surfaces of section for $\xi_{\|}=0.1, \xi_{\perp}=0.001$, and two different energies, $E=10^{-4}$ (Figs. 5a,b), and $10^{-3}$ (Figs. 5c,d).
The left panels refer to the Poincaré sections when $\epsilon=0$, while the right panels refer to the same sections when $\epsilon=10^{-5}$. In the case of Fig. 5b, the energy $E=10^{-4}$ is of the order of $\epsilon^{2} / \xi_{\perp}^{2}$. Thus, magnetic forces are the same order of magnitude as electric forces. This means that the electric field can be considered as introducing a large perturbation to the system. This effect causes a considerable deformation of the invariant curves on the surface of section with respect to the same curves when $\epsilon=0$ (Figs. 5a,b). The position of the central periodic orbit is also shifted considerably.

For larger energies, the particles' velocities are large enough so that electric forces are small compared to magnetic forces. In this case the surfaces of section are very similar with or without the electric field (Figs. 5c,d), except that the whole section is displaced by an effective displacement $z=\epsilon / \xi_{\perp}^{2}$ for $\epsilon \neq 0$. In particular, similar phenomena occur as regards the separation of the regular and chaotic domains and the chaotic escapes along arcs. However, the set of invariant curves are displaced by $z=\epsilon / \xi_{\perp}^{2}$ for $\epsilon \neq 0$.

\subsection{The limit of a strong longitudinal field $\xi_{\|}$: electrons}

At the limit of a strong longitudinal field $\xi_{\|} \rightarrow 1$ the dynamics of electrons tends to the limit of the guiding center approximation. This is due to the fact that, for a typical (close to thermal) velocity of electrons $v \sim 10^{-2}$, the gyroradius both at the center and at the edges of the sheet becomes of order $R_{\mathrm{g}} \sim 10^{-2}$, i.e., it is much smaller than the characteristic scale length of the sheet. Formally, the guiding center approximation 

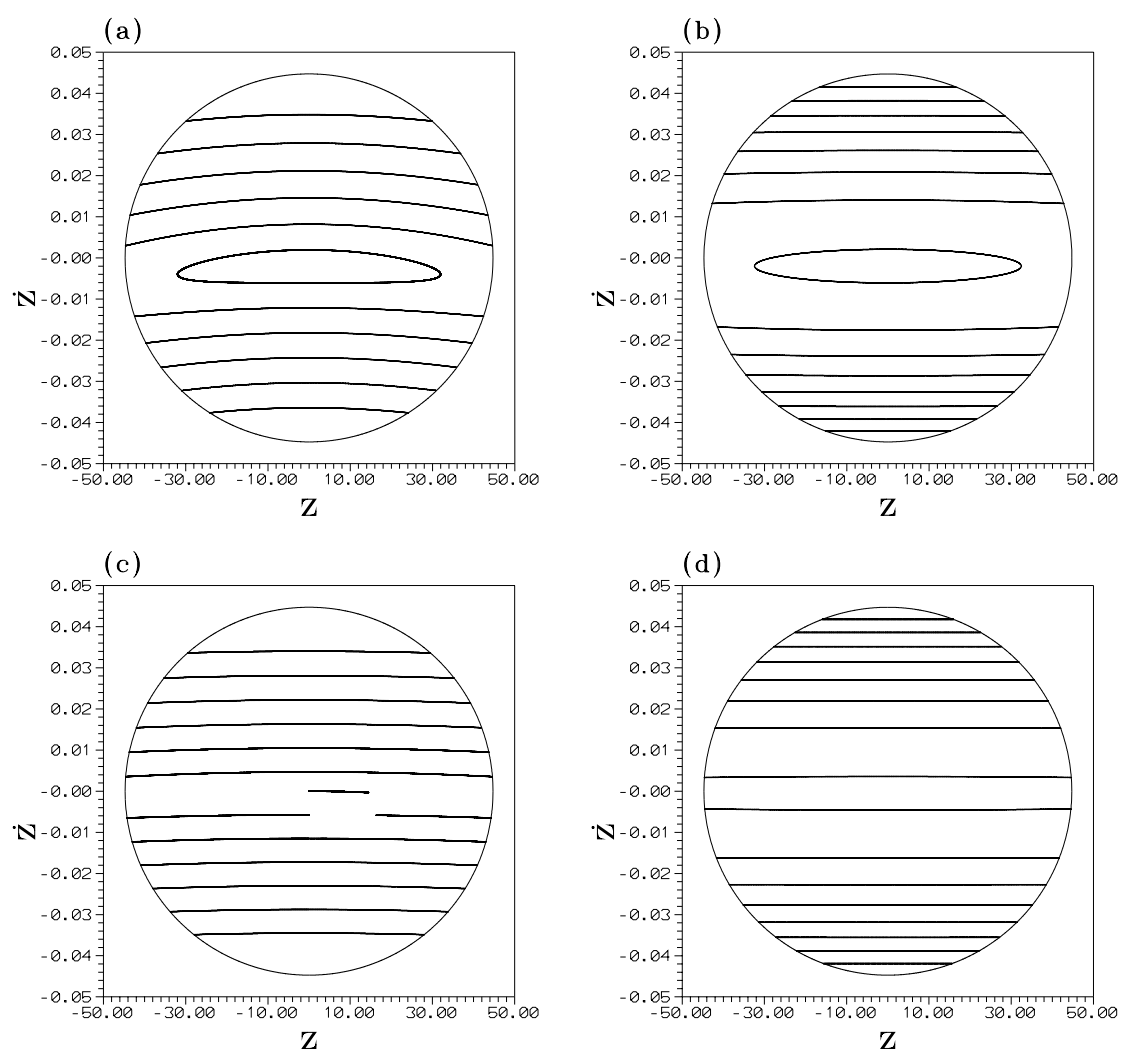

Fig. 6. a) The Poincaré surface of section $(z, \dot{z}), y=0, \dot{y}>0$, derived by numerical integration of orbits in the Hamiltonian (21), for the energy $E=10^{-3}$ for the values $\xi_{\perp}=0.001$, and a) $\xi_{\|}=0.5$, c) $\xi_{\|}=1$. The corresponding theoretical invariant curves, derived by the adiabatic approximation (Eq. (46)) are shown in b) and d) respectively.

corresponds to considering only the lowest order term $Z_{2}$ in the normal form (Eq. (32)) at the limit $\xi_{\|} \gg \xi_{\perp}$. Thus we consider the Hamiltonian

$Z=\xi_{\|} J_{1}+\frac{1}{2}\left(p_{2}^{\prime 2}+\omega_{2}^{2}(J) u_{2}^{2}\right)$

where the following approximations are made:

$J_{1}=\frac{1}{2 \xi_{\|}}\left(\xi_{\|}^{2} u_{1}^{2}+p_{1}^{2}\right), \quad \omega^{2}\left(J_{1}\right)=\frac{\xi_{\perp}^{2} J_{1}}{\xi_{\|}^{3}}, \quad p_{2}^{\prime} \simeq p_{2}+J_{1} / 2 \xi_{\|}$

and

$u_{1} \simeq-y+\frac{\xi_{\perp}}{\xi_{\|}} z, \quad p_{1} \simeq-p_{y}+\frac{\xi_{\perp}}{\xi_{\|}} c_{4}, \quad u_{2} \simeq z, \quad p_{2} \simeq c_{4}$.

For an orbit of energy $Z=E$, the adiabatic invariant $J_{1}$ can be expressed in terms of the variables $\left(u_{2}, p_{2}\right) \simeq\left(z, c_{4}\right)$ by making the corresponding substitutions of variables in Eq. (46) and finding the positive root with respect to $J_{1}$. One finds:

$$
\frac{J_{1}^{2}}{4 \xi_{\|}^{2}}+\left(2 \xi_{\|}+\frac{p_{2}}{\xi_{\|}}+\frac{u_{2}^{2} \xi_{\perp}^{2}}{\xi_{\|}^{3}}\right) J_{1}+\left(p_{2}^{2}-2 E\right)=0 .
$$

The family of curves $\left(u_{2}, p_{2} ; E, J_{1}\right)$ given by Eq. (47), for constant parameters $\left(E, J_{1}\right)$, are invariant ellipses on the $\left(u_{2}, p_{2}\right)$ plane. At the maximum value of $J_{1}$, the corresponding ellipse shrinks to an invariant point. The maximum of $J_{1}$ on the plane $\left(u_{2}, p_{2}\right)$ is at the point

$u_{2}=0, \quad p_{2}=-\frac{E}{2 \xi_{\|}^{2}}, \quad J_{1, \max }=\frac{E}{\xi_{\|}}$.
For $y=0$ we have $u_{2} \simeq z, p_{2} \simeq \dot{z}$. Thus, the Poincaré surface of section $(z, \dot{z})$ for $y=0, \dot{y}>0$, for constant $E$, corresponds to the parts of the invariant ellipses (47) where the corresponding orbit has intersections with the plane $y=0$. These parts are limited by the limiting curve $\dot{y}=0$ (Eq. (39)). Figure 6 shows the comparison between the theoretical invariant curves predicted by (Eq. (47)) and the precise invariant curves obtained by numerical integration of electron orbits on the Poincaré section $(z, \dot{z})$ for $y=0, \dot{y}>0$, for a constant value of the energy $E=10^{-3}$. The field components are $\xi_{\perp}=0.001$, while $\xi_{\|}$has the value 0.5 in Figs. $6 a, b$, and 1 in Figs. $6 c$,d. We note that, as $\xi_{\|}$increases, the ellipses become so elongated (because $\omega\left(J_{1}\right)$ in Eq. (46) is of order $\left.\omega\left(J_{1}\right)=O\left(\xi_{\|}^{-3}\right)\right)$ that their parts within the limiting curve of the Poincaré section become elongated arcs approaching straight horizontal segments (Figs. 6c,d). This explains why many invariant curves on the surface of section are open.

If we now turn on the electric field $\epsilon$, the dynamics is described quite accurately by adding an electrostatic potential term to the lowest order terms of the normal form (Eq. (46)), namely:

$Z=\xi_{\|} J_{1}+\frac{1}{2}\left(p_{2}^{\prime 2}+\omega_{2}^{2}(J) u_{2}^{2}\right)-\epsilon u_{2}$

where the variables $J_{1}, p_{2}^{\prime}, u_{2}$ are given in terms of the original variables by the same formulae as in Eq. (46).

The Hamiltonian (49) yields integrable orbits consisting of two independent oscillations. In particular, similarly to the case 
(a)

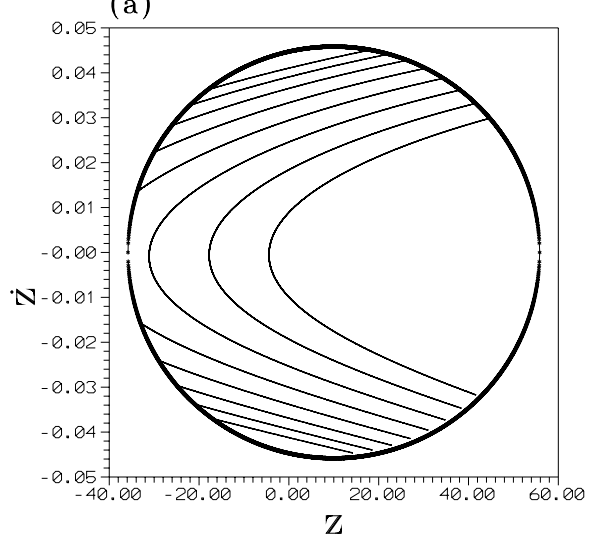

(b)

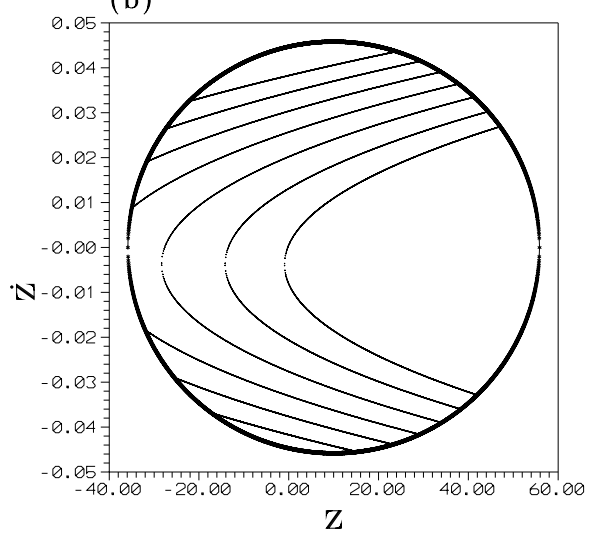

Fig. 7. Poincaré surfaces of section $(z, \dot{z}), y=0, \dot{y}>0$, for the Hamiltonian (20), with $\xi_{\|}=1, \xi_{\perp}=0.001, \epsilon=10^{-5}$ and $E=10^{-3}$. a) The section derived by numerical integration of the orbits b) Theoretical invariant curves which correspond to segments of invariant ellipses given by Eq. (49).

$\epsilon=0$, the projection of an orbit in the plane $\left(u_{2}, p_{2}\right)$, for constant values of $E \equiv Z$ and $J_{1}$, lies on an invariant ellipse which is the integral curve of a formal integral of motion. However, the center of an ellipse is shifted with respect to the position $u_{2} \simeq z=0$. The approximation $y \simeq-u_{1}$ made in the previous subsection is no longer valid, because the orbit undergoes oscillations with a large amplitude in the $z$-axis, so that the term $\xi_{\perp} z / \xi_{\|}$is dominant in the transformation $y=-u_{1}+\xi_{\perp} z / \xi_{\|}$. However, the form of the Hamiltonian (49) allows one to extract directly the shift of the center of the ellipse in the $z$-axis:

$z_{c}=\frac{\epsilon}{\omega^{2}\left(J_{1, \max }\right)}=\frac{\epsilon \xi_{\|}^{4}}{\xi_{\perp}^{2} E}$.

In view of the constancy of the integral $I_{2}$ (Eq. (18)), the above equation implies a drift with constant average velocity $\dot{x}=-\xi_{\perp} z_{c}$ in the $x$-axis. Thus, the centre of the family of ellipses does not correspond to a real periodic orbit, but only to an orbit which is periodic in its $(y, z)$ projection, while it drifts along the magnetic field lines in the $x$-direction.

Figure 7 shows the Poincaré surface of section $(z, \dot{z}), y=$ $0, \dot{y}>0$ for electrons with initial kinetic energy $E=10^{-3}$, as derived by direct numerical integration of the orbits, when the field components are $\xi_{\perp}=10^{-3}, \xi_{\|}=1$ and $\epsilon=10^{-5}$ (Fig. 7a). Figure 7b gives the theoretical invariant curves as derived from Eq. (49), for the same energy $E=Z$ and for different values of $J_{1}$. The main remark is that the shift predicted by Eq. (50), namely $z_{c}=10000$, is three orders of magnitude bigger than the shift of the center of the limiting curve (Eq. (43)), i.e., $\epsilon / \xi_{\perp}^{2}=10$. Thus, the Poincaré section $y=0$ contains arcs which are segments of the outermost left part of the invariant ellipses given by Eq. (49). The intersection of these arcs with the limiting curve marks the starting and ending points of the segments of an orbit that have recurrent intersections with the plane $y=0$.

\subsection{The limit of a strong longitudinal field $\xi_{\|}$: protons}

For equal values of the kinetic energy, the gyroradius of a proton orbit is larger than the gyroradius of an electron orbit by a factor $\simeq 45$. Thus, for protons at thermal velocities we have $R_{\mathrm{g}} \sim 0.45$, i.e., the gyroradius is a significant fraction of the current sheet width. This implies that typical proton orbits cannot be described by the guiding center approximation.

On the other hand, the analysis made by the normal form (Eq. (34)) continues to be precise. Figure 8 shows the projections $y(t), z(t)$ and $u_{1}(t)$ for a typical proton orbit with energy $E=0.5$. The main difference of proton orbits, relative to electron orbits is in the much larger values of the action $J_{1}$, which leads to oscillations of the $u_{1}$ normal variable with amplitude close to unity. This means that higher order terms of the normal form are required to describe accurately the dynamics. For example, the frequency of oscillations in Fig. $8 \mathrm{c}$ is equal to $\omega_{1}=1.14>\omega_{10}=\xi_{\|}=1$. If the term $c\left(J_{1}^{\prime}\right)$ in the normal form (34) is taken into account, one finds $\omega_{1}=\omega_{10}+\partial c / \partial J_{1}^{\prime}$. Setting $J_{1}^{\prime} \simeq 1$ yields the theoretical estimate $\omega_{1} \simeq 1.12$, which is quite close to the numerically determined frequency.

Now, for $\epsilon \neq 0$, the dynamics for protons is determined by the facts that a) the value of the action $J_{1}$ is large $\left(J_{1} \geq 1\right)$, and b) the dimensionless parameter $\epsilon$ of the electric field is three orders of magnitude greater than that of electrons for the same value of the electric field. The consequence of (a) is that the values of $u_{1}$ are large. The consequence of (b) is that the electric potential term $-\epsilon u_{2} \simeq-\epsilon z$ is the dominant term in the normal form (49), up to quite large values of $z$.

These effects are demonstrated in Fig. 9, where a typical proton orbit, with initially thermal velocity, is plotted up to the moment when the proton escapes from the sheet $(y=1$, at $t \simeq 1050$, Fig. 9a). The fact that the $-\epsilon z$ term is dominant in the Hamiltonian is demonstrated by comparing the $z(t)$ projection of the orbit with a theoretical constant acceleration term $z(t)=$ $\epsilon t^{2} / 2$ corresponding to the action of a potential $-\epsilon z$.

In view of the fact that $u_{1}$ is now a large quantity, an inversion of the normalizing transformation $z=\xi_{\|}\left(y-u_{1}\right) / \xi_{\perp}$ yields $u_{1} \simeq \xi_{\perp} z / \xi_{\|}$, i.e., the function $u_{1}(t)$ is modulated by the function $z(t)$ times a factor $\xi_{\perp} / \xi_{\|}$. This is clearly visible in a comparison of Figs. 9b,c. Furthermore, the oscillatory part of both $y(t)$ and $u_{1}(t)$ shows segments with non-constant oscillation period, meaning that the corresponding orbit is weakly chaotic. 

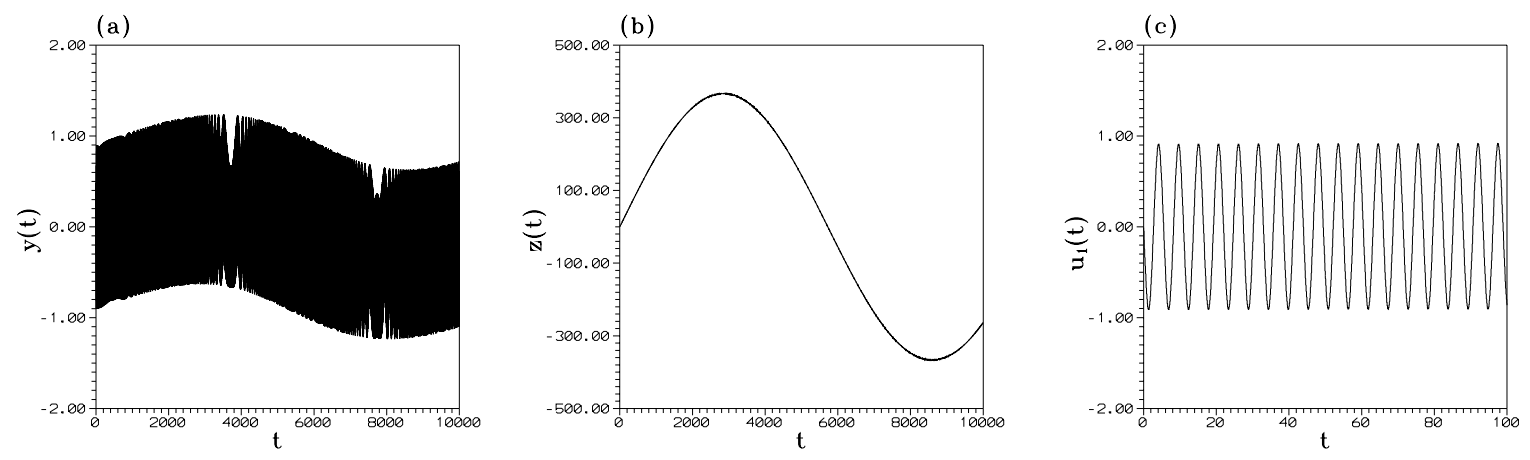

Fig. 8. A typical proton orbit in the Hamiltonian (21), for the values $\xi_{\perp}=0.001, \xi_{\|}=1$, and energy $E=0.5$. a) $y(t)$, b) $z(t)$, and c) $u_{1}(t)$.
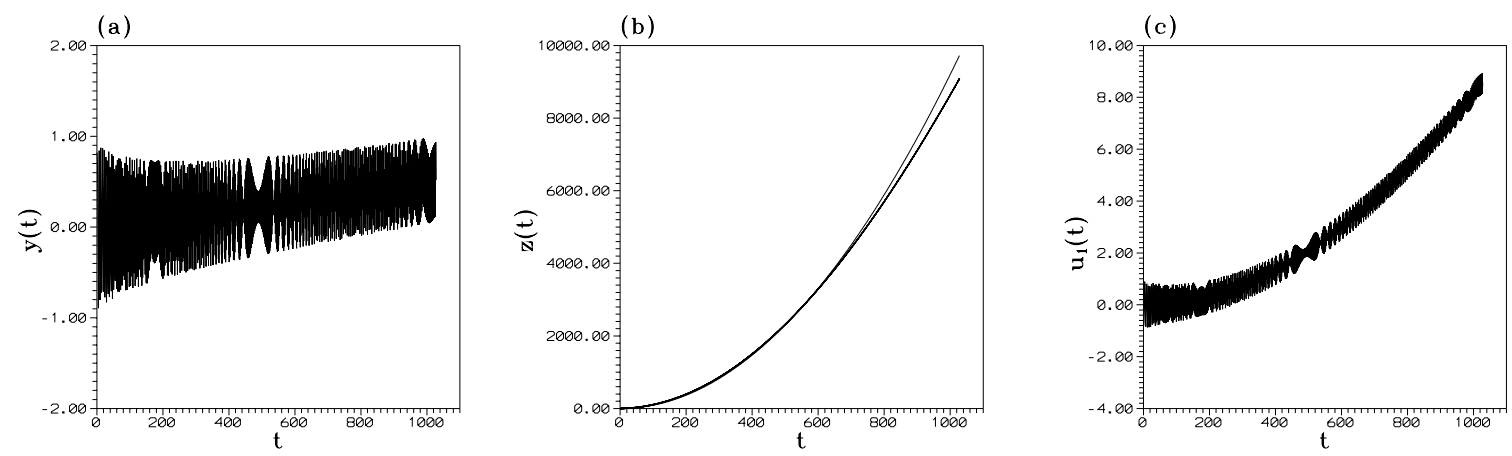

Fig. 9. A typical proton orbit in the Hamiltonian (20), for the values $\xi_{\perp}=0.001, \xi_{\|}=1, \epsilon=1.8 \times 10^{-2}$ and energy $E=0.5$. a) $y(t)$, b) $z(t)$, and c) $u_{1}(t)$. The soft solid line in $\left.\mathbf{b}\right)$ is a theoretical curve $z(t)=\frac{1}{2} \epsilon t^{2}$.

In conclusion, for a strong value of the longitudinal field $\xi_{\|}$, typical proton orbits are weakly chaotic, and they are effectively described by a constant acceleration term in the direction of the electric field.

\section{Maximum acceleration length and kinetic energy gain}

This section studies numerically the kinetic energy gain for electrons and protons along escaping orbits injected at the edges of the current sheet. The results of the previous section can be exploited in order to derive analytical formulae that are compared with the results of the numerical simulations.

As demonstrated above, protons escape mostly along weakly chaotic orbits. Electrons escape along either regular or chaotic orbits, depending on the value of the longitudinal field $\xi_{\|}$. When $\xi_{\|}$is of the order of the main reconnecting component, the electrons follow regular orbits preserving an adiabatic invariant which is described by the normal form theory of Sect. 4 . On the other hand, if $\xi_{\|}$is not very strong $\left(\xi_{\|} \leq 10^{-1}\right)$, the electrons follow weakly chaotic orbits, and the escape is along manifolds in phase space which intersect the Poinaré surface of section at the arcs shown in Fig. 5d.

Now, chaotic orbits are free to explore the whole energetically available phase space up to the moment of escape, since they are not limited by a third integral of motion. Thus, the maximum possible kinetic energy gain along chaotic orbits, as a function of the initial kinetic energy of an orbit, is determined only by the outer boundary of the curves of zero velocity at the limiting planes $|y|=y_{\text {out }}$. For any value of $I_{2}$, this is given by

$\xi_{\perp}^{2} z^{2}-2\left(\xi_{\perp} I_{2}+\xi_{\|} \xi_{\perp} y+\epsilon\right) z+I_{2}^{2}+2 I_{2} \xi_{\|} y+\xi_{\|}^{2} y^{2}=2 E$

which is the generalization of Eq. (42) for a non-zero value of $I_{2}$. Solving Eq. (51) for $z$, we find the $z$ limits of the zero velocity curve when the particle crosses the edges $y_{\text {out }}$ of the sheet.

$$
\begin{aligned}
z_{\min , \max }= & \frac{1}{\xi_{\perp}^{2}}\left(\xi_{\perp} I_{2}+\xi_{\|} \xi_{\perp} y_{\mathrm{out}}+\epsilon\right. \\
& \left. \pm \sqrt{2 \xi_{\perp} I_{2} \epsilon+2 \xi_{\|} \xi_{\perp} y_{\mathrm{out}} \epsilon+\epsilon^{2}+2 \xi_{\perp}^{2} E}\right)
\end{aligned}
$$

In our simulations, $y_{\text {out }}= \pm 1$ and, without loss of generality, injections are from $z=0$. The value of $I_{2}$ is determined by the value of $y=y_{\text {in }}$ at the injection point, and the component $\dot{x}$ of the initial velocity (which does not explicitly appear in the equations of motion). The starting point is set slightly below the edge of the sheet $\left(y_{\text {in }}= \pm 0.9\right)$. This is because the gyroradious for typical electron orbits at $y=1$ is $R \simeq 0.1$, and we wish to avoid counting as escaping particles that intersect the edge along their first gyration. Finally, $I_{2}$ is set to the value $I_{2}=-\xi_{\|} y_{\text {in }}= \pm 0.09$, in order to keep the initial value of the velocity component $\dot{x}$ equal to $\dot{x}=0$ (see Eq. (21)).

Independently of the details of an orbit, the maximum kinetic energy gain, as a function of the initial kinetic energy $E_{0}$, is calculated by setting the maximum acceleration length equal to $z_{\max }$, and $E=E_{0}$. Thus

$\Delta E_{\max }=\epsilon z_{\max }$. 


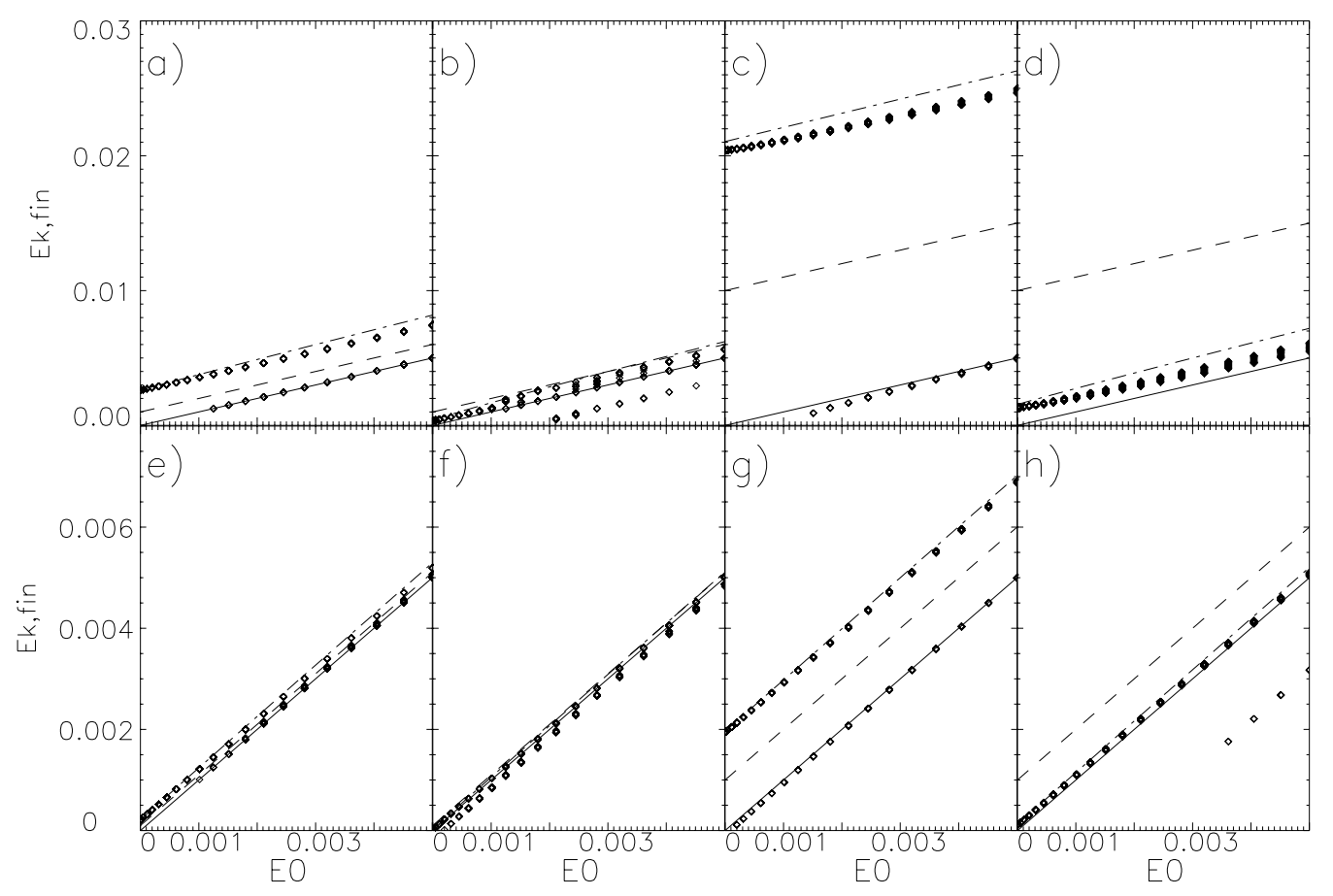

Fig. 10. The final kinetic energy vs. the initial kinetic energy for electrons in escaping orbits, for various parameters of the RCS and initial conditions of the orbits. The energy unit is $5.66 \times 10^{3} \mathrm{keV}$. The solid line is the diagonal. A dashed curve corresponds to a theoretical formula of Litvinenko (1996) (Eq. (55)). The upper dot-dashed curve corresponds to a theoretical calculation of the maximum kinetic energy gain (Eq. (52) below). The electric field is $\epsilon=10^{-5}$. a) $\xi_{\perp}=10^{-3}, \xi_{\|}=10^{-1}, y_{\text {in }}=-0.9$, b) $\xi_{\perp}=10^{-3}, \xi_{\|}=10^{-1}, y_{\text {in }}=0.9$, c) $\xi_{\perp}=10^{-3}, \xi_{\|}=1, y_{\text {in }}=-0.9$, d) $\xi_{\perp}=10^{-3}, \xi_{\|}=1, y_{\text {in }}=0.9$, e) $\left.\xi_{\perp}=10^{-2}, \xi_{\|}=10^{-1}, y_{\text {in }}=-0.9, \mathbf{f}\right) \xi_{\perp}=10^{-2}, \xi_{\|}=10^{-1}, y_{\text {in }}=0.9$, g) $\xi_{\perp}=10^{-2}, \xi_{\|}=1, y_{\text {in }}=-0.9$, h) $\xi_{\perp}=10^{-2}, \xi_{\|}=1, y_{\text {in }}=0.9$.

Escapes from the upper boundary $y_{\text {out }}=1$ are possible for all values of the initial kinetic energy $E_{0}$. On the other hand, escapes from the boundary $y_{\text {out }}=-1$ are only possible when $E_{0}$ is large enough. We readily find

$E_{0} \geq \frac{\xi_{\|} \epsilon}{\xi_{\perp}}\left(1-\frac{\epsilon}{2 \xi_{\perp} \xi_{\|}}-\frac{I_{2}}{\xi_{\|}}\right)$

from Eq. (52). In the latter case, the energy gain is smaller compared to that of escapes from the upper boundary, due to the term $-\xi_{\|} \xi_{\perp} \epsilon$ in the square root term of Eq. (54). Furthermore, Eq. (52) implies that for a certain choice of parameter values it is possible that $z_{\min }<0$. Thus, it is possible that particles in chaotic orbits escape by losing rather than gaining energy. This happens when a particle leaves the sheet at a point with $z$ - coordinate close to the limiting value $z=z_{\min }$.

These phenomena are seen in Figs. 10, 11, which show the final kinetic energy as a function of the initial kinetic energy for escaping particles (electrons in Fig. 10, protons in Fig. 11) injected at both edges of the sheet, for various characteristic values of the magnetic field components $\xi_{\|}, \xi_{\perp}$. The diamonds correspond to orbits injected with various angles in the range 0 to $\pi$ pointing towards the sheet interior. Proton orbits are obtained with the same initial conditions as electron ones, by changing the value of the dimensionless electric field as explained in Sect. 2.

The theoretical upper bound to the amount of possible kinetic energy gain (Eqs. (51) and (53)) is shown by a dot-dashed line. Note that many escaping electrons (Fig. 10) are below the diagonal (solid line in all panels) meaning that they escape by losing rather than gaining kinetic energy. Finally, due to the preservation of the integral $I_{2}=-\xi_{\|} y_{\text {in }}$, injections from $y_{\text {in }}=-0.9$ (Figs. 10a,c,e,g and $11 \mathrm{a}, \mathrm{c}, \mathrm{e}, \mathrm{g}$ ) have always larger energy gains than injections from $y_{\text {in }}=0.9$ (Figs. 10b,d,f,h and $11 \mathrm{~b}, \mathrm{~d}, \mathrm{f}, \mathrm{h})$. Physically, the accelerated particle spends more time in the sheet in the former case than in the latter case.

Finally, a comparison is made to existing formulae in the literature. A dashed line in each panel corresponds to the energy gain following Litvinenko (Eq. (34) in Litvinenko 1996):

$z_{\max }=\frac{\xi_{\|}}{\xi_{\perp}}, \Delta E=\frac{\epsilon \xi_{\|}}{\xi_{\perp}}$

This formula is an approximation for orbits that can be treated by a perturbative technique. This formula can be recovered by a precise treatment in the case of regular orbits following the guiding center approximation. Namely, the equation of the invariant ellipses (49) and the normalizing transformation of Eq. (46) yields, at the limit $J_{1} \ll 1, y \simeq \xi_{\perp} z / \xi_{\|}$. Thus, along any invariant ellipse given by Eq. (49), the escaping value $y=y_{\text {out }}=1$ corresponds to an acceleration length $z \simeq \xi_{\|} / \xi_{\perp}$, i.e., a kinetic energy gain $\Delta E \simeq \epsilon \xi_{\|} / \xi_{\perp}$. However, a crossing of the opposite plane $y=-1$ leads to escapes through a loss of kinetic energy, since $z \simeq-\xi_{\|} / \xi_{\perp}, \Delta E \simeq-\epsilon \xi_{\|} / \xi_{\perp}$.

Independently of the regular or chaotic nature of an orbit, Eq. (55) corresponds also to the limiting case of Eqs. (52), (53) when $\epsilon \ll \xi_{\|} \xi_{\perp}$ and $I_{2}=0, E_{0} \rightarrow 0$. These conditions are 


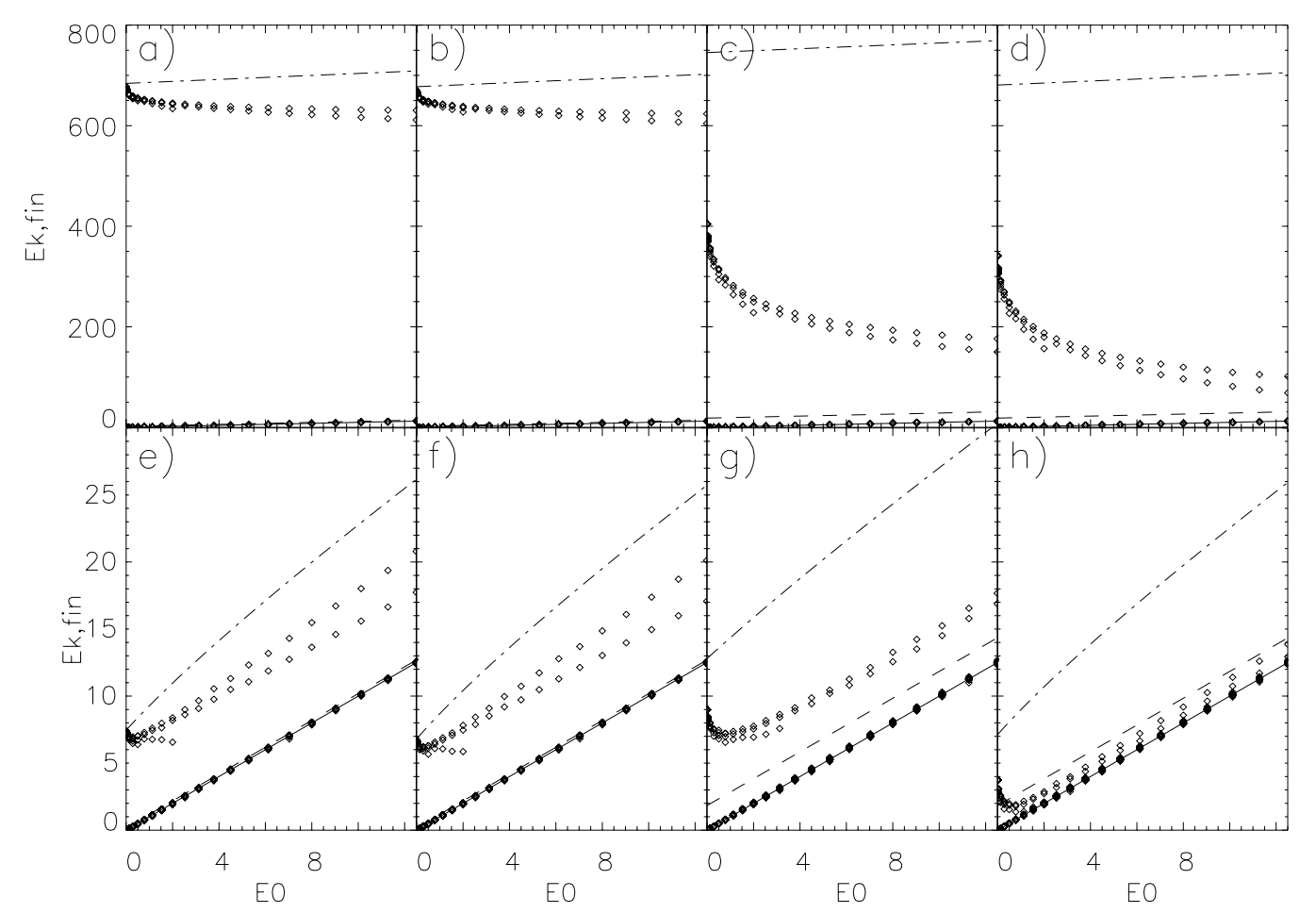

Fig. 11. The final kinetic energy vs. the initial kinetic energy for protons in escaping orbits, for various parameters of the RCS and initial conditions of the orbits. The energy unit is $3.087 \times 10^{-3} \mathrm{MeV}$. The solid and dashed lines, as well as the parameters of the magnetic field are as in Fig. 4. The electric field parameter is $\epsilon=1.84 \times 10^{-2}$.

likely to be valid for electrons. Note that since $I_{2}=0$, the initial conditions of orbits in Eq. (55) should be considered to be at $y_{\text {in }}=0$. Thus, as shown in Figs. 10a,c,e,g, Eq. (55) corresponds to half the total kinetic energy gain for particles injected at $y_{\text {in }}=$ -0.9 .

The situation is quite different for protons (Fig. 11). There, most escaping orbits are weakly chaotic. Equations (52), (53) provide an upper limit of the kinetic energy gain, which, however, is only order of magnitude correct. Furthermore, the condition $\epsilon>\xi_{\|} \xi_{\perp}$ typically holds. At the limit of large $\epsilon$, Eqs. (53) or (52) tend asymptotically to the limiting formula

$$
\Delta E \simeq \frac{2 \epsilon^{2}}{\xi_{\perp}^{2}}
$$

i.e., one recovers asymptotically Speiser's (1965) formula (see also Heerikhuisen et al. 2002 for a relativistic correction).

\section{Conclusions}

In this paper, the orbits of charged particles (electrons and protons), under the influence of the magnetic and electric fields inside a Harris-type reconnecting current sheet (Fig. 1), are studied by dynamical systems methods. The emphasis is on building an approximate analytical theory giving the approximate integrals of regular orbits and the domains of chaotic orbits. The kinetic energy gain of escaping particles is analytically determined as a function of the physical parameters of the RCS and of the initial conditions of the particles' orbits. The analytical results are compared to results obtained by numerical integration of representative orbits. The following are our main conclusions:

1) The problem of stability for motions perpendicular to the plane of reconnection is shown to correspond to a problem of "parametric resonance". It is shown that, for all values of the electric field $\epsilon$, zones of instability exist for arbitrarily large values of the longitudinal magnetic field $\left(\xi_{\|}\right)$. The width of these zones decreases as the value of the longitudinal field increases. The stability criterion given by Litvinenko and Somov (1993) is satisfactory for quite large values of $\epsilon$.

2) The equations of motion can be reduced to Hamilton's equations in a two degrees of freedom Hamiltonian dynamical system. The orbits are studied analytically by transforming the 2DOF Hamiltonian to a normal form. Accurate formulae are given for the orbits obeying an "adiabatic invariant". These orbits define invariant curves on appropriate Poincaré surfaces of section. The orbits in chaotic domains escape by a Fermi-type chaotic scattering mechanism.

3) For moderate values of the longitudinal magnetic field $\left(\xi_{\|} \sim 10\right.$ percent of the main reconnecting component), both electrons and protons escape along weakly chaotic orbits subject to Fermi-type acceleration. However, for large values of $\xi_{\|}$(of the same order as the main reconnecting component or larger), electrons escape through regular orbits. The passage of electrons from the RCS is represented by segments of ellipses on the Poincaré surface of section. On the other hand, protons' orbits are quite similar to constant acceleration orbits.

4) The kinetic energy gains of escaping particles are determined numerically as a function of the type of particle (electron or proton), the strengths of the magnetic field components 
$\xi_{\perp}, \xi_{\|}$and the initial kinetic energy at injection in the current sheet. The resuts are in good agreement with the above analytical predictions.

5) Useful formulae are derived, relating the maximum kinetic energy gain $\Delta E$ of a particle escaping the sheet to the physical parameters of the sheet and the initial kinetic energy $E_{0}$ upon injection. The main formulae are Eq. (53) for a maximum acceleration length given by (52). The asymptotic formula $\Delta E=\epsilon \xi_{\|} / \xi_{\perp}$ (Litvinenko 1996) holds for the kinetic energy gain of electrons from the center of the sheet to the escape point, when the initial kinetic energy $E_{0}$ is small. For large $E_{0}$, $\Delta E$ scales as $\Delta E \propto E_{0}^{1 / 2}$. For protons, the same equations lead asymptotically to Speiser's formula $\Delta E=2 \epsilon^{2} / \xi_{\perp}^{2}$ for large $\epsilon$. A number of particles may leave the sheet with a net loss of kinetic energy. This phenomenon is explained theoretically.

Acknowledgements. This research was supported in part by a research grant of the Academy of Athens. We would like to thank Professor G. Contopoulos for many helpful discussions and Dr. G. Petrie for a careful reading of the manuscript. A.A. wishes to thank Prof. V. Zharkova for a private communication that helped clarify a particular point of the present paper. C.G. devotes this work to the memory of Minas Gontikakis, a continuous source of inspiration.

\section{Appendix}

\subsection{Calculation of the Normal Form with Lie series}

The Lie series method introduces canonical transformations as mappings $q, p \rightarrow q^{\prime}, p^{\prime}$ induced by the Hamiltonian flow for $t=1$ under a Hamiltonian $\chi$ called "generating function". Any function $F(q, p)$ of the old canonical variables is transformed to a function $F\left(q^{\prime}, p^{\prime}\right)$ of the new canonical variables according to

$F\left(q^{\prime}, p^{\prime}\right)=\exp \left(L_{\chi}\right) F(q, p)$

where $L_{\chi}=\{\cdot, \chi\}$ is the Poisson bracket operator of the function $\chi$, and the exponential operator $\exp \left(L_{\chi}\right)$ is defined as

$\exp \left(L_{\chi}\right)=\sum_{k=0}^{\infty} \frac{1}{k !} L_{\chi}^{k}$.

For a given non-integrable Hamiltonian $H(q, p)$, the normal form $Z$ is a transformation of $H$ to new variables, obtained by the composition of $r$ successive Lie canonical transformations with generating functions $\chi_{3}, \chi_{4}, \ldots, \chi_{r}$, where $\chi_{s}$ is of order $s$ in the canonical variables. The transformed Hamiltonian after $r$ steps, denoted $H^{(r)}$ is in normal form up to terms of order $r$, namely

$$
\begin{aligned}
H^{(r)} & \equiv \exp \left(L_{\chi_{r}}\right) \exp \left(L_{\chi_{r-1}}\right) \ldots \exp \left(L_{\chi_{3}}\right) H \\
& =Z_{2}+Z_{3}+Z_{4}+\ldots Z_{r}+h_{r+1}^{(r)}+\ldots
\end{aligned}
$$

( $H^{(2)}$ corresponds to the original Hamiltonian).

Let the quadatic part of the Hamiltonian $\mathrm{H}_{2}$ be given by

$H_{2} \equiv \frac{1}{2}\left(p_{x}^{2}+\omega_{1}^{2} x^{2}\right)+\frac{1}{2} p_{y}^{2}$.

By a canonical transformation

$q_{1}=\frac{X_{1}-i P_{1}}{\sqrt{2}}, \quad p_{1}=\frac{P_{1}-i X_{1}}{\sqrt{2}}$ with $X_{1} \equiv \sqrt{\omega_{1}} x, P_{1} \equiv p_{x} / \sqrt{\omega_{1}}$ the Hamiltonian $H_{2}$ is written as

$H_{2}=i \omega_{1} p_{1} q_{1}+\frac{p_{y}^{2}}{2}$.

The recursive scheme which defines the generating function $\chi_{r}$ at order $r$ is determined by the equation

$D_{\omega} \chi_{r}-h_{r}^{(r-1)}+Z_{r}=0$

where $D_{\omega}$ denotes the linear operator

$D_{\omega}=\left\{\cdot, H_{2}\right\}$

and $Z_{r}$ denotes the normal form at order $r$. The function $Z_{r}$ is the sum of a number of monomial terms of the form $q_{1}^{k_{1}} p_{1}^{l_{1}} y^{k_{2}} p_{y}^{l_{2}} . Z_{r}$ is integrable if the following definition is used:

Definition 1: a monomial term $q_{1}^{k_{1}} p_{1}^{l_{1}} y^{k_{2}} p_{y}^{l_{2}}$ is said to be in normal form with respect to the operator $D_{\omega}$ if $k_{1}=l_{1}$, and $l_{2}=0$.

The action of $D_{\omega}$ on any monomial $q_{1}^{k_{1}} p_{1}^{l_{1}} y^{k_{2}} p_{y}^{l_{2}}$ is given by $D_{\omega} q_{1}^{k_{1}} p_{1}^{l_{1}} y^{k_{2}} p_{y}^{l_{2}}=i\left(k_{1}-l_{1}\right) \omega_{1} q_{1}^{k_{1}} p_{1}^{l_{1}} y^{k_{2}} p_{y}^{l_{2}}+k_{2} q_{1}^{k_{1}} p_{1}^{l_{1}} y^{k_{2}-1} p_{y}^{l_{2}+1}$.

Thus, if the normal form terms $Z_{r}$ are chosen by

$Z_{r}=$ terms of $h_{r}^{(r-1)}$ satisfying Definition 1

Eq. (63) is reduced to the form

$D_{\omega} \chi_{r}-\tilde{h}_{r}^{(r-1)}=0$

where

$\tilde{h}_{r}^{(r-1)}=h_{r}^{(r-1)}-Z_{r}$.

Now, Eq. (67) can be written as a linear system of algebraic equations for the unknown coefficients of all the possible monomial terms included in $\chi_{r}$. Considering the set $\mathcal{A}_{k l}$ which includes all the terms of $\tilde{h}_{r}^{(r-1)}$ of the form:

$$
\begin{array}{r}
\mathcal{A}_{k l}=\left\{a_{k l, n} q_{1}^{k} p_{1}^{l} q_{2}^{n} p_{2}^{r-k-l-n}: k+l \leq r,\right. \\
n=0,1, \ldots, r-k-l\}
\end{array}
$$

the coefficients $a_{k l, n}$, for all $k, l, n$ with $k+l+n=r$ are known. Let $b_{k l, n}$ be the unknown coefficients of the same monomial terms in the function $\chi_{r}$. Taking into account Eqs. (67) and (65), the two sets of coefficients are linked by a bidiagonal linear system of equations

$$
\left(\begin{array}{ccccc}
\lambda & 1 & & & \\
& \lambda & 2 & & \\
& & \ddots & \ddots & \\
& & & \lambda & r-k-l \\
& & & & \lambda
\end{array}\right)\left(\begin{array}{c}
b_{k l, 0} \\
b_{k l, 1} \\
\vdots \\
\vdots \\
b_{k l, r-k-l}
\end{array}\right)=\left(\begin{array}{c}
a_{k l, 0} \\
a_{k l, 1} \\
\vdots \\
\vdots \\
a_{k l, r-k-l}
\end{array}\right),
$$

where $\lambda=i(k-l) \omega_{1} \neq 0$ (since the terms of $\mathcal{A}_{k l}$ do not belong to the normal form. Equation (70) has a unique solution

$b_{k l, n}=\sum_{m=n}^{r-k-l} \frac{m !}{n !} \frac{a_{k l, m}}{\lambda^{m-n+1}}$. 
All the coefficients $b_{k l, n}$ of $\chi_{r}$ are thus specified. Once $\chi_{r}$ is specified, the Lie series transformation of the Hamiltonian $H^{(r)}=\exp \left(L_{\chi_{r}}\right) H^{(r-1)}$ is calculated. This completes one iteration of the normal form algorithm.

\section{References}

Anastasiadis, A., Vlahos, L., \& Georgoulis, M. 1997, ApJ, 489, 367 Anastasiadis, A., Gontikakis, C., Vilmer, N., \& Vlahos, L. 2004, A\&A, 422, 323

Arnold, V. I. 1978, Mathematical Aspects of Classical Mechanics, (Springer-Verlag)

Arnold, V. I., \& Novikov, S. P. 1995, Dynamical Systems VII, Encyclopaedia of Mathematical Sciences, Vol. 16 (SpringerVerlag)

Birkhoff, G. D. 1927, Dynamical Systems, Amer. Math. Soc., (Providence, R.I.)

Browning, P. K., \& Vekstein, G. E. 2001, J. Geophys. Res., 106, 18677

Bruhwiler, D. L., \& Zweibel, E. G. 1992, J. Geophys. Res., 97, 10825

Büchner, J., \& Zelenyi, L. M. 1989, J. Geophys. Res., 94, 11821

Büchner, J., \& Zelenyi, L. M. 1991, Adv. Space Res., 11, 177

Bulanov, S. V. 1980, Sov. Astron. Lett., 6, 206

Burkhart, G. R., Drake, J. F., \& Chen, J. 1990, J. Geophys. Res., 95, 18833

Chen, J. 1992, J. Geophys. Res., 97, 15011

Chen, J., \& Palmadesso, P. J. 1986, J. Geophys. Res., 91, 1499

Chirikov, B. V. 1979, Phys. Rep. 52, 263

Contopoulos, G., \& Vlahos, L. 1975, J. Math. Phys., 16, 1469

Contopoulos, G. 2002, Order and Chaos in Dynamical Astronomy, (Springer)

Cowley, S. W. H. 1978, Planet. Space Sci., 26, 539

Craig, I. J. D., \& Litvinenko, Y. 2002, ApJ, 570, 387

Dalla, S., \& Browning, P. K. 2005, A\&A, 436, 1103

Deeg, H. J., Borovsky, J. E., \& Duric, N. 1991, Phys. Fluids B, 3, 2660

Dragt, A. J., \& Finn, J. M. 1979, J. Math. Phys., 20, 2649

Engel, U. M., Stegemerten, B., \& Eckelt, P. 1995, J. Phys. A, 28, 1425
Fletcher, L., \& Petkaki, P., 1997, Sol. Phys., 172, 267

Fletcher, L., Pollock, J. A., \& Potts, H. E. 2004, Sol. Phys., 222, 279

Hagyard, M. J. 1990, it Mem. S.A. It., 61, 337

Hamilton, B., McClements, K. G., Fletcher, L., \& Thyagaraja, A. 2003, Sol. Phys., 214, 339

Hamilton, B., Fletcher, L., McClements, K. G., \& Thyagaraja, A. 2005, ApJ, 625, 496

Heerikhuisen, J., Litvinenko, Y. E., \& Craig, I. J. D. 2002, ApJ, 566, 512

Ince, E. L. 1926, Ordinary Differential Equations (London: Dover)

Kliem, B. 1994, ApJS, 90, 719

Lichtenberg, A. J., \& Lieberman, M. A. 1992, Regular and Chaotic Dynamics (Springer-Verlag)

Litvinenko, Y. E., \& Somov, B. V. 1993, Sol. Phys., 146, 127

Litvinenko, Y. E. 1993, Sol. Phys., 147, 337

Litvinenko, Y. E. 1996, ApJ, 462, 997

Martens, P. C. H. 1988, ApJ, 330, 131

Martens, P. C. H., \& Young, A. 1990, ApJS, 73, 333

Mori, K. I., Sakai, J. I., \& Zhao, J. 1998, JpS., 494, 430

Moses, R. W., Finn, J. M., \& Ling, K. M. 1993, J. Geophys. Res., 98, 4013

Nodes, C., Birk, G. T., Lesch, H., \& Schopper, R. 2003, Phys. Plasmas, 10,835

Petkaki, P., \& MacKinnon, A. L. 1997, Sol. Phys., 172, 279

Poincaré, H., 1892, Les Méthodes Nouvelles de la Mécanique Céleste (Paris: Gauthier-Villars)

Priest, E. R., \& Forbes, T. 2000, Magnetic Reconnection, MHD Theory and Applications (Cambridge University Press)

Somov, B. V. 1992, Physical Processes in Solar Flares (Kluwer)

Speiser, T. W. 1965, J. Geophys. Res., 70, 4219

Speiser, T. W., \& Lyons, L. R. 1984, J. Geophys. Res., 89, 147

Vekstein, G. E., \& Browning, P. K. 1997, Phys. Plasmas, 4, 2261

Vlahos, L., Isliker, H., \& Lepreti, F. 2004, ApJ, 608, 540

Zharkova, V., \& Gordovskyy, M. 2004, ApJ, 604, 884

Zharkova, V., \& Gordovskyy, M. 2005, MNRAS, 356, 1107

Zhu, Z., \& Parks, G. 1993, J. Geophys. Res., 98A, 7603 